\title{
What is new in computer vision and artificial intelligence in medical image analysis applications
}

\author{
Jimena Olveres ${ }^{1,2}$, Germán González ${ }^{2}$, Fabian Torres ${ }^{1,2}$, José Carlos Moreno-Tagle ${ }^{2}$, \\ Erik Carbajal-Degante ${ }^{3}$, Alejandro Valencia-Rodríguez ${ }^{4}$, Nahum Méndez-Sánchez ${ }^{4,5}$, Boris Escalante- \\ Ramírez $^{1,2} \wedge$
}

${ }^{1}$ Centro de Estudios en Computación Avanzada, Universidad Nacional Autónoma de México (UNAM), Mexico City, Mexico; ${ }^{2}$ Departamento de Procesamiento de Señales, Facultad de Ingeniería, UNAM, Mexico City, Mexico; ${ }^{3}$ Posgrado en Ciencia e Ingeniería de la Computación, UNAM, Mexico City, Mexico; ${ }^{4}$ Unidad de Investigación en Hígado, Fundación Clínica Médica Sur, Mexico City, Mexico; ${ }^{5}$ Facultad de Medicina, UNAM, Mexico City, Mexico

Contributions: (I) Conception and design: J Olveres, N Méndez-Sánchez, B Escalante-Ramírez; (II) Administrative support: J Olveres, B EscalanteRamírez; (III) Provision of study materials or patients: All authors; (IV) Collection and assembly of data: All authors; (V) Data analysis and interpretation: All authors; (VI) Manuscript writing: All authors; (VII) Final approval of manuscript: All authors.

Correspondence to: Boris Escalante-Ramírez. Centro de Estudios en Computación Avanzada CECAV. Universidad Nacional Autónoma de México. 3er piso, Torre de Ingeniería, Cd. Universitaria, Mexico City, C.P. 04510, Mexico. Email: boris@unam.mx.

\begin{abstract}
Computer vision and artificial intelligence applications in medicine are becoming increasingly important day by day, especially in the field of image technology. In this paper we cover different artificial intelligence advances that tackle some of the most important worldwide medical problems such as cardiology, cancer, dermatology, neurodegenerative disorders, respiratory problems, and gastroenterology. We show how both areas have resulted in a large variety of methods that range from enhancement, detection, segmentation and characterizations of anatomical structures and lesions to complete systems that automatically identify and classify several diseases in order to aid clinical diagnosis and treatment. Different imaging modalities such as computer tomography, magnetic resonance, radiography, ultrasound, dermoscopy and microscopy offer multiple opportunities to build automatic systems that help medical diagnosis, taking advantage of their own physical nature. However, these imaging modalities also impose important limitations to the design of automatic image analysis systems for diagnosis aid due to their inherent characteristics such as signal to noise ratio, contrast and resolutions in time, space and wavelength. Finally, we discuss future trends and challenges that computer vision and artificial intelligence must face in the coming years in order to build systems that are able to solve more complex problems that assist medical diagnosis.
\end{abstract}

Keywords: Artificial intelligence (AI); computer vision (CV); medical image analysis; cardiology; oncology; microscopy; neurodegenerative disorders; respiratory diseases; gastroenterology

Submitted October 13, 2020. Accepted for publication Apr 20, 2021.

doi: 10.21037 /qims-20-1151

View this article at: http://dx.doi.org/10.21037/qims-20-1151

^ ORCID: Jimena Olveres, 0000-0002-1514-4520; Boris Escalante-Ramírez, 0000-0003-4936-8714. 


\section{Introduction}

Undoubtedly, it is intangible to think about all the technological advances that have been developed in the field of medicine in recent decades. Not only have they allowed us to understand more precisely the anatomy and physiology of the different organs that structure the human body, but they have also let us advance in the identification and, therefore, the treatment of several diseases from very early stages in different areas of medicine. This has been largely accomplished by the development of computer vision (CV) and artificial intelligence (AI). Briefly, these tools provide us the ability to acquire, process, analyze, and understand an infinite number of static and dynamic images in real time, which will represent a better characterization of each disease, and a better patient selection for early interventions.

Since many diagnostic methods available to date present the disadvantage of being invasive, expensive and/or very complex for their standardization in most parts of the world, assisted diagnosis through $\mathrm{CV}$ and $\mathrm{AI}$ represent a feasible solution that allows to identify a broad number of different diseases from initial stages, define better the treatment and follow-up, and decrease the health care costs associated with each patient.

The union of high-performance computing with machine learning (ML) offers the capacity to deal with big medical image data for accurate and efficient diagnosis. Moreover, AI and CV may reduce the significant intra- and inter-observer variability, which undermines the significance of the clinical findings. AI allows to automatically make quantitative assessments of complex medical image with increased diagnosis accuracy.

The objective of this review is to assess in an understandable and well-structured way recent advances in automatic medical image analysis in some of the diseases with higher incidence and prevalence rates worldwide.

Cardiovascular diseases (CVD), oncological disorders and pulmonary diseases such as chronic obstructive pulmonary disease (COPD) and coronavirus disease 2019 (COVID-19) have positioned themselves as the main causes of mortality in individuals 50 years of age and older (1-5). Moreover, CVD are also the main cause of premature death (4). Gastrointestinal and liver diseases account for some of the highest burden and cost in public health care worldwide $(4,6)$. Moreover, the lack of timely attention at early stages of these diseases lead to high morbidity and mortality rates (3). Neurodegenerative diseases such as Alzheimer's and Parkinson's have increased their incidence in modern times. As life expectancy increases, so does the occurrence of these diseases (7). Although AI has contributed to develop a large number of methods that assist the diagnosis of these diseases $(8,9)$, we limit this review to $\mathrm{CV}$ and $\mathrm{ML}$ methods applied on medical imaging, namely ultrasound, computer tomography, magnetic resonance, microscopy and dermoscopy. We show how these methods are used to detect, classify, characterize and enhance relevant information in order to aid clinical diagnosis and treatment.

In the next section, the relation of medical imaging with $\mathrm{CV}$ and $\mathrm{AI}$ is discussed from a general point of view. Subsequent sections show recent advances and applications of these two areas of computer science that have improved diagnosis performance of the above-mentioned diseases.

\section{$C V$ and $A I$ in medical imaging}

According to Patel et al. (10) some of the earliest works on AI applied to medicine date from the 1970s when AI was an already known discipline and the term was created at the very famous 1956 Dartmouth College conference (11). While many researchers created modelbased image processing based on AI, they did not call them that, even when their algorithms were from this area (10). Since then, new applications started to emerge that led to the first conference of the new organization Artificial Intelligence in Medicine Europe (AIME), at Maastricht, The Netherlands on June 1991, that established the term $\mathrm{AI}$ in Medicine. At first, most methods were based on expert systems. More recently, Kononenko (12) punctuates that the goal of AI in medicine is to try to make computers more intelligent and one of the basic requirements of intelligence is the ability to learn. ML appeals to that concept and some of the first successful algorithms in medicine were naive Bayesian approaches (12).

CV deals with a large range of problems such as image segmentation, object recognition, detection, reconstruction, etc. It aims at modeling and understanding the visual world by extracting useful information from digital images, often inspired by complex tasks of human vision. Although it exists since the 1960s, it remains an unsolved and challenging task to the extent that only recently computers have been able to provide useful solutions in different application fields. It is a multidisciplinary subject closely related to AI. AI is a broad area of computer science that 
aims at building automatic methods to solve problems that typically require human intelligence. ML, in turn, is a part of AI that builds systems that are able to automatically learn from data and observations. Most successful methods of $\mathrm{CV}$ have been developed with ML techniques. Among the most widely used methods of ML are Support Vector Machines (SVM), Random Forests, Regression (linear and logistic), K-Means, k-nearest neighbors (k-NN), Linear Discriminant Analysis, Naive Bayes (NB), etc.

Deep learning (DL) is a subfield of ML that has become

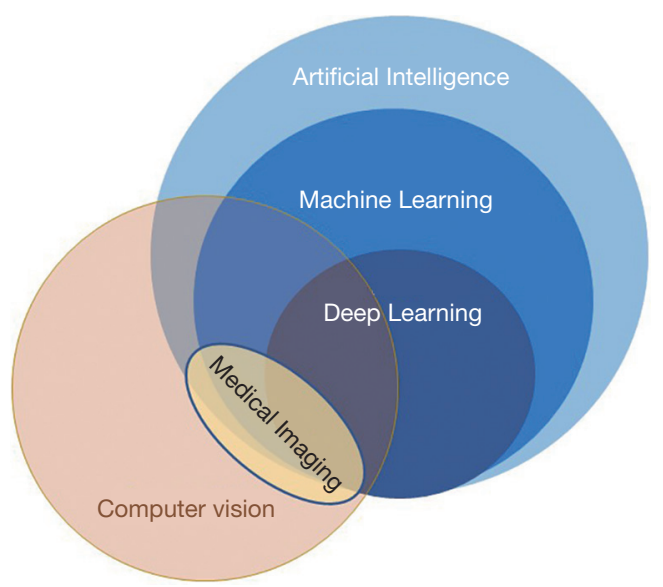

Figure 1 Relation between computer vision and artificial intelligence. an attractive and popular tool in $\mathrm{CV}$ because of its amazing results in complex problems of visual information analysis and interpretation. The term deep refers to multiple-layer neural networks models. In recent years, there has been a rising interest in applying DL models to medical problems (12-14). For example, deep neural networks have shown amazing results in skin lesion classification tasks. Some outstanding examples can be found in annual challenges (15).

Examples of DL models are convolutional neural networks (CNNs), recurrent neural networks, long shortterm memory, generative adversarial networks, etc. Recent advances in this field have shown impressive accuracies and measured results. A schematic relation between $\mathrm{CV}$ and $\mathrm{AI}$ in medical imaging is presented in Figure 1.

The interaction between $\mathrm{CV}$ and $\mathrm{AI}$ in medical imaging is shown in Figure 2. Relevant feature extraction in medical image databases is a first critical step to train a new ML model. The training process aims at obtaining a model that has learned a specific task such as segmentation, classification, detection, recognition, etc. on the specific training data. Next, the model is tested with new input data that undergoes the same feature extraction process. The results of the task achieved with this test data are evaluated with performance metrics. If results do not meet the user requirements, the process is repeated until a new combination of feature extraction and ML methods satisfies the required performance level.

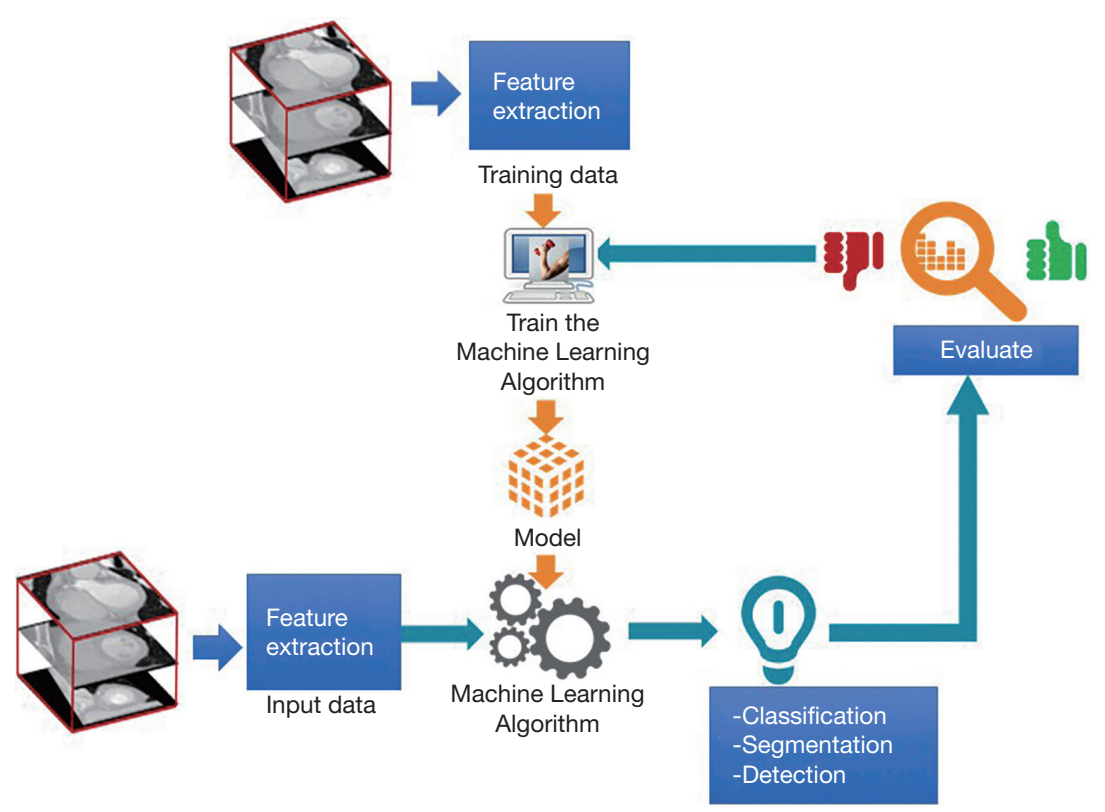

Figure 2 The flow of computer vision and machine learning interaction in medical imaging 


\section{CVD}

Heart analysis is relevant for understanding cardiac diseases which are a major concern in the health area. They constitute a multifactorial and multidisciplinary clinical disease, leading to a great detriment to the quality of patients' life. The World Heart Federation reports CVD as the main cause of morbidity and mortality in almost two thirds of the world population. Some of the most important diseases are related to the heart and blood vessels. They include coronary heart diseases, cerebrovascular disease, peripheral arterial disease, rheumatic heart disease, congenital heart disease, deep vein thrombosis and pulmonary embolism. Behavioral risk factors such as unhealthy diet, physical inactivity, alcohol consumption, and obesity represent major risk factors for heart failure (16).

Different medical imaging modalities are used for the assessment of heart failure such as computed tomography (CT), magnetic resonance imaging (MRI) and echocardiography (EG). Clinical analysis of these images comprises qualitative as well as quantitative examinations.

Applications of AI aiming at assessing heart function have ranged from localizing and segmenting anatomical structures to recognizing structural as well as dynamic patterns, and more recently to automatically identifying and classifying several diseases. Whenever heart failure occurs, the heart shows a reduced function. This may cause the left ventricle (LV) to lose its ability to contract or relax normally. In response, LV compensates for this stress by modifying its behavior, which creates hypertrophy and progresses to congestive heart failure (17). These patterns can be recognized and evaluated by AI.

Anatomical and dynamical parameter estimations are necessary to assess heart failure. These tasks related with imaging technology are so relevant that in the last decades their research and development allowed not only to search for better resolution and contrast in their studies, but also the possibility to merge anatomical and functional information. Moreover, new imaging protocols have been developed to comply with analysis and segmentation tasks. Chen et al. presented a complete study of different DL techniques in various image modalities to segment one, two or all cardiac structures (18), as well as coronary arteries, all of them supporting clinical measurements such as volume and ejection fraction.

Some of the more difficult problems of automatic segmentation include poor image quality, low contrast and poor structure border detection. Moreover, cardiac anatomy and gray value distribution of the images varies from person to person, especially when a cardiopathy exists. If we add the intra vendor and scanners differences, the challenge for the development of more sophisticated tools is considerable.

Many different methods for ventricle segmentation have been reported in the literature, where classical active contours are some of the more popular (19). CNNs often excel because of its improved segmentation performance despite their dependence on large amounts of training data (18). This is a serious issue in the case of the medical area, because of data availability restrictions in clinical environments and because of the lack of medical experts willing to annotate large amounts of data. This is a tedious task, causing fatigue as well as intra and inter variability between experts (20). In some areas such as CT and MRI challenges have emerged with annotated databases $(18,21,22)$.

The use of DL has become common not only for cardiac segmentation but also to support classic algorithms, for example when searching for an initial localization of the cardiac structures to segment. Recently, this initialization has been achieved with a $\mathrm{CNN}$ that finds a coarse shape while a level set refines the contour, as shown on Figure 3. This hybrid method has the advantage of using only a small database to train a neural network, e.g., a UNet, since only a rough initial segmentation is needed; detail refining is a task left to the active contour method

Different neural network models emerged for segmentation tasks, such as the UNet model or even Full Convolutional Networks (FCN) as the main algorithm $(23,24)$. These methods have proved to obtain good results. Table 1 presents some of the relevant works in the cardiovascular area with a mention to the imaging modality.

\section{Motion analysis as support for heart failure analysis}

Most 2D segmentation methods analyze complete volumes on a slice by slice basis losing inter-slice interactions. They usually fail in some challenging slices located on the base or on the apex where contours are not well defined. To address these problems additional information, such as motion, has been introduced in many of these methods. Optical flow estimation comprises techniques that can be used to provide information of cardiac motion through the cardiac cycle. Dynamic changes of the cardiac walls can be characterized by motion vectors showing the heart deformation. Heart's motion estimation can assist the study of different malformations and anomalies, and this information can be enhanced again with different image features. Over the 


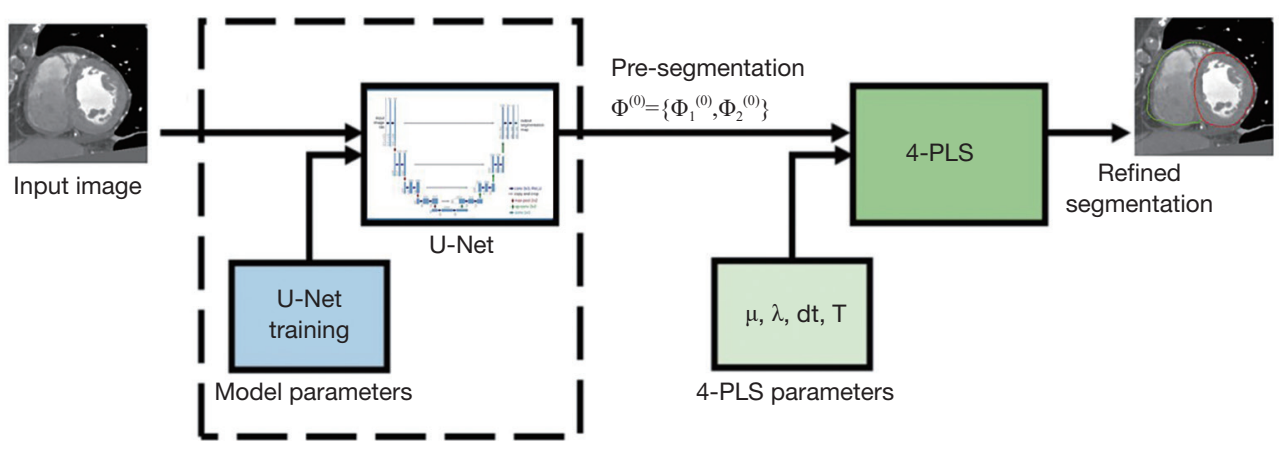

Figure 3 Modules of a segmentation step using UNet deep learning as initialization.

Table 1 Cardiovascular applications. Selection of relevant works of different methods in cardiovascular area with different image modalities

\begin{tabular}{|c|c|c|c|}
\hline Selected works & Description & Type of images & Year \\
\hline Petitjean et al. (19) & Short axis view segmentation with active contours method & MR & 2011 \\
\hline Carbajal-Degante et al. (23) & CNN and active contours for segmentation & CT & 2020 \\
\hline Zhang et al. (25) & Creation of a CNN based tool that identifies cardiopathies in clinical practice & Echocardiogram & 2018 \\
\hline
\end{tabular}

MR, magnetic resonance; CT, computer tomography; US, ultrasound.

years, the study of cardiac movement has been carried out mainly in two dimensional images, however, it is important to consider that the deformations due to the movement of the heart occur in a three-dimensional space. New 3D results can demonstrate the heart's movement complexity as shown in Figure 4 (26).

Future trends in the cardiac area aim at building fully automatic tools able to understand the images and relate them to specific pathologies. This is the case of Zhang et al. (25), who developed a complete tool that identifies different cardiopathies. Their integrated system analyses echocardiogram studies and is composed of modules that start from identifying metadata from Digital Imaging and Communications in Medicine format, followed by a pipeline of different DL algorithms with four tasks, (I) image classification, (II) image segmentation, (III) measurements of cardiac structure and function, and (IV) disease detection. Decisions are taken during the pipeline, ending in a differentiation of patients' characteristics that indicate a disease detection, such as hypertrophic cardiomyopathy or pulmonary hypertension.

\section{Oncological diseases}

\section{Breast ultrasound (BUS)}

Breast cancer has become the most frequent cancer and the number one cause of cancer-related deaths among women around the world, with rates increasing in nearly every region globally. Early detection and screening are critical to improve the prognosis for the patient. Screening consists in identifying cancers before any symptoms appear and, although, mammography is recommended by the WHO for breast cancer screening, ultrasound has become a useful tool for assisting and complementing this procedure, especially in limited resource settings with weak health systems where mammography is not cost-effective (27). While mammography has a high sensitivity in the detection of microcalcifications, ultrasound is able to detect tumors in dense breast tissue, usually found in women under age 30, with higher sensitivity than mammography (28). Young women have low incidence rates of breast cancer; however, its incidence increases at a faster rate than in older women and their tumors tend to be larger, with a higher grade of 

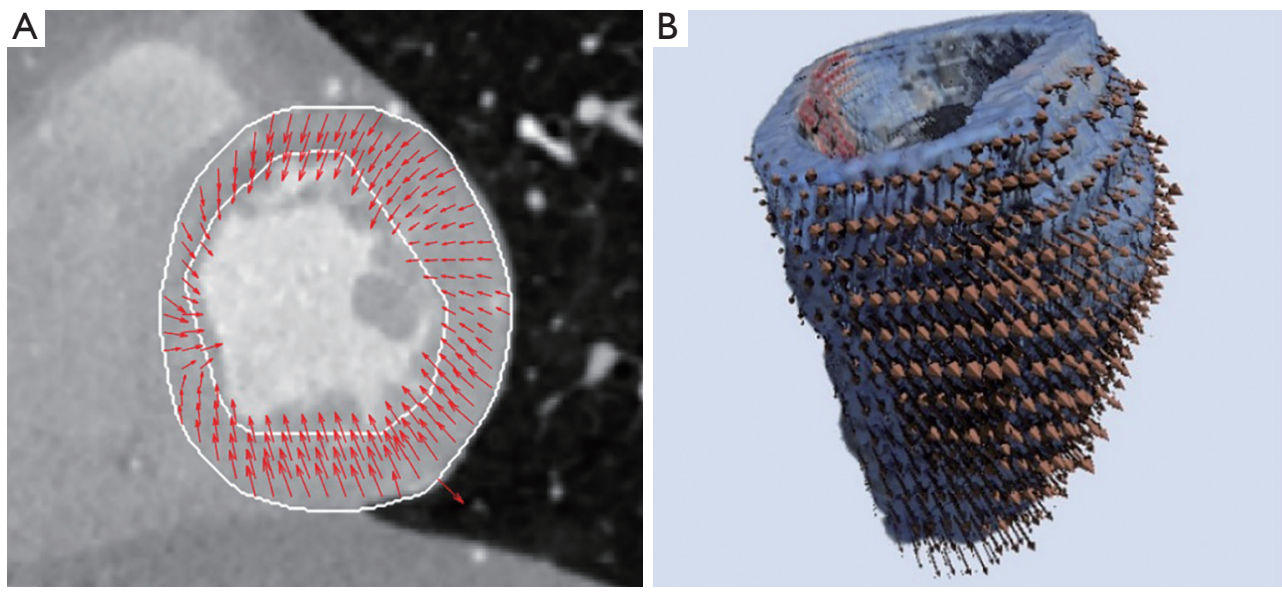

Figure 4 Motion estimation. (A) 2D motion estimation results for heart left ventricle on systole, short axis view. (B) Heart volume with 3D motion estimation results on diastole.

malignancy and poorer prognostic characteristics, making ultrasound necessary as a complementary technique in breast screening. Also, ultrasound is the best imaging modality to identify if the lesion is solid, such as a benign fibroadenoma or cancer, or a fluid-filled benign cyst (29). Even with this advantage, the visualization of lesions in BUS images is a difficult task due to some intrinsic characteristics of the images like speckle, acoustic shadows and blurry edges (30). These drawbacks make ultrasound imaging and diagnosis highly operator-dependent and with a high inter-observer variation rate, increasing the risk of oversight errors. Therefore, computer-aided diagnosis (CAD) systems are desirable to help radiologists in breast cancer detection and classification. CAD systems using BUS images generally focus on solving three problems: lesion detection, lesion segmentation, and lesion classification.

\section{Lesion detection and segmentation}

Accurate automatic detection and segmentation methods of breast tumors can help the experts to achieve faster diagnoses, and it's a key stage of fully automatic systems for breast cancer diagnosis using ultrasound images (31). Because of the mentioned inherent artifacts in BUS images the automatic detection and segmentation of lesions in this imaging modality is not an easy task (30).

Ultrasound gray-level intensities provide helpful information about the density of different tissues found in the images, however the poor quality of ultrasound images due to speckle noise makes the differentiation difficult. Because of this, most of the proposed methods for tumor detection and segmentation in BUS images use a pre-processing step to obtain more homogenous regions and enhance the contrast of the image, while preserving important diagnostic features $(32,33)$. The internal echo pattern of structures has been used to depict the local variation of pixel intensities to detect abnormal regions within the BUS images, to distinguish lesion regions from normal tissue, acoustic shadows and glands. Texture information seems highly suitable for characterizing internal echo patterns in different structures. However, texture analysis in ultrasound images is not an easy task and many metrics have been used to describe the echo patterns in breast tumors (34-36).

Several studies report automatic methods (30-40), where modeling the knowledge of BUS and oncology as prior constraints is needed. Based on the literature, ML-based methods stand out among the most popular. Supervised methods like AdaBoost, Artificial Neural Networks and Random Forest are the most common methods used for lesion detection and segmentation in BUS (37). Image features used in these methods should be appropriately selected according to the application, and texture information seems highly suitable for ultrasound images, but making an appropriate Feature Selection is an important step specially when dealing with high dimensionality spaces (30). Feature selection methods like Principal Component Analysis and Genetic algorithms have been used to improve the results in the detection and segmentation of lesions, with true positive fraction (TPF) values of $84.48 \%$; however, these approaches still rely on manually designated features, which depend on good understanding of the 
Table 2 Lesion detection and segmentation. Selection of relevant works for lesion detection and segmentation in oncological diseases

\begin{tabular}{lll}
\hline Selected works & Description & Techniques \\
\hline Chen et al. (30) & Semi-automatic 3D lesion segmentation method & Discrete active contours with automatic initialization \\
Moon et al. (31) & Automatic 3D lesion detection & 3D mean shift and fuzzy c-means clustering \\
Madabhushi et al. (32) & Automatic 2D lesion segmentation & Directional gradient deformable shape-based model \\
and probabilistic classification of image pixels & 2003 \\
Liu et al. (35) & Automatic 2D lesion detection & Kernel SVM and classification checkpoints \\
Torres et al. (38) & Automatic 2D lesion detection & $\begin{array}{l}\text { Genetic algorithms for feature and parameter selection 2019 } \\
\text { and random forest classification }\end{array}$ \\
Yap et al. (39) & Automatic 2D lesion segmentation & Patched and fully convolutional CNNs \\
Huang et al. (41) & Automatic 3D lesion segmentation method & Neural networks applied to 2D texture patches \\
\hline
\end{tabular}

SVM, Support Vector Machines; CNN, convolutional neural network.

images and the lesions (38). DL, which is a new field of ML, can directly learn abstract levels of features directly from images, solving the need to propose an initial set of features to describe the problem. CNNs such as LeNet, UNet and FCN-AlexNet, have been successfully implemented in the tasks of lesion detection and segmentation with TPF values of $89.0 \%, 91.0 \%$ and $98.0 \%$ (39) respectively. A summary of representative works can be seen in Table 2.

\section{Classification of breast lesions}

The malignancy of a tumor is estimated by the expert ultrasonographer mainly from its shape, echogenicity and the internal echo pattern. Morphological features such as roundness, aspect ratio, convexity and compactness have been used to characterize the shape of tumors. These descriptors along with texture analysis have shown good results in the classification of breast lesions using ML approaches (40). Most of the proposed methods used classical ML approaches including Linear Regression, Fuzzy Logic Systems and SVM, where the main difference between them are the chosen descriptors used to characterize lesions with accuracy values up to 95.45\% (37). CNNs have been used for the classification of lesions by producing a set of transformation functions and image features directly from the data to characterize the malignancy of the lesion. Different CNN models, including GoogleNet, ResNet50, Inception V3, Xception and Mask-RCNN have been proposed for this task, having accuracy values up to $98.0 \%$ for the classification of breast lesions into benign and malignant $(42,43)$. A summary of representative works can be seen in Table 3. Figure 5 shows a segmentation and classification of two different breast lesions.

\section{Fundamental issues and future directions}

Although CV and AI methods have been successfully applied for breast lesion detection, segmentation, and classification, there are some fundamental issues that need to be solved before fully CAD systems can be developed taking advantage of these techniques. In fully automatic methods a key step is the lesion detection, which delivers candidate regions of interest (ROIs). Although ML approaches have shown good results in this task, it remains an open and challenging problem because the appearance of benign and malignant lesions are quite different and the quality of the images will also depend on the ultrasonic device, contributing to the difficulty of modeling the lesions. Another important aspect that should be considered is the presence of posterior acoustic shadowing, which appears as a dark region behind the lesion, often obfuscating lesion margins and making it difficult to determine the shape of the lesion. Since this is a challenging issue, this artifact has been overlooked in many BUS CAD systems, however there is an urgent to overcome this problem in order to improve these systems (44). Moreover, the increasing use of $3 \mathrm{D}$ ultrasound imaging calls for new lesion detection, segmentation and classification techniques that can be used in a $3 \mathrm{D}$ surgical context $(41,45)$. For the development of these techniques, the creation of new datasets that consider ethical considerations is necessary (46). 

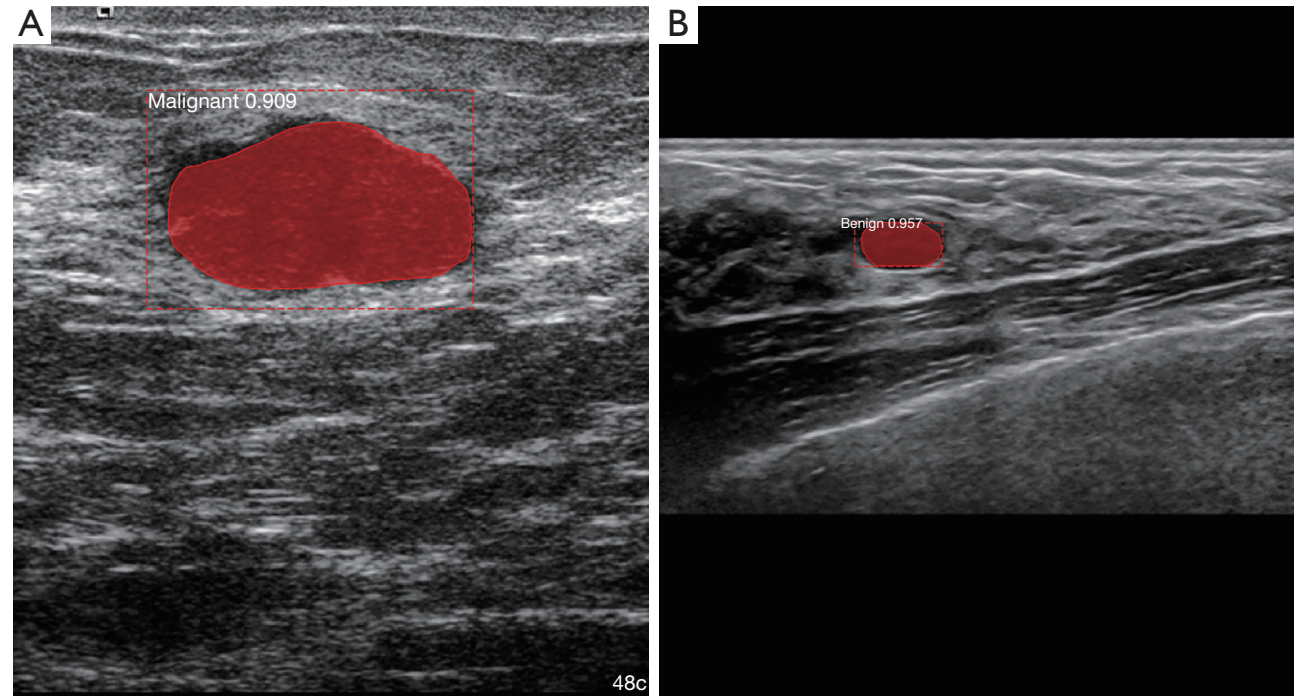

Figure 5 Detection, segmentation, and classification of breast lesions in US using Mask R-CNN. (A) Malignant lesion. (B) Benign lesion.

Table 3 Breast lesion analysis. Selection of relevant works for detection and classification of breast lesions

\begin{tabular}{lll}
\hline Selected works & Description & Year \\
\hline Chen et al. (36) & Texture classification of 3D ultrasound breast using run difference matrix with neural networks & 2005 \\
Cao et al. (42) & $\begin{array}{l}\text { Evaluation of the performance of several state-of-the-art DL model for the detection and classification for } \\
\text { breast lesions }\end{array}$ \\
Chiao et al. (43) & Automatic detection, segmentation and classification of breast lesions using Mask RCNN \\
\hline
\end{tabular}

$\mathrm{DL}$, deep learning.

\section{Deep learning in dermatology}

The skin is the largest organ in the human body (47) while skin cancer is the most common form of cancer in the United States (U.S.) (48). According to the American Academy of Dermatology, it is estimated that 1 in 5 Americans will develop skin cancer in their lifetime. As with any other type of cancer, the sooner it is spotted, the better the prognosis becomes. When skin cancer is caught early, it is highly treatable. This type of cancer is mainly diagnosed through a visual inspection. Among the different types of malignant neoplasia, melanoma is a rare form of skin cancer that develops from skin cells called melanocytes which are located in the layer of basal cells at the deepest part of the epidermis, they are also found in the iris, inner ear, nervous system, heart and hair follicles among other tissues. Melanoma represents fewer than $5 \%$ of all skin cancers in the U.S. However, melanoma is also the deadliest form of skin cancer, it is responsible for about $75 \%$ of all skin cancer related deaths (49). In Mexico, melanoma is responsible for $80 \%$ of deaths from skin cancer (50). It is increasing in the world, more than any other malignancy. According to the latest report of the National Institute of Cancerology (Instituto Nacional de Cancerología, INCan), the number of cases in Mexico increased $500 \%$ in recent years (51).

Dermatologists use different visual inspection methods to determine if a skin lesion under analysis may be melanoma (malignant). Some of these methods are the ABCDE rule, 7 points checklist, and the Menzies method (52) just to name a few. All these methods are based on the visual features that make a malignant melanoma lesion distinguishable. Dermatology is a medical specialty which can greatly benefit from the powerful feature extraction capabilities of deep neural networks. 
Table 4 Skin lesion classification. Selection of relevant works of deep learning methods applied to skin lesion detection and classifications

\begin{tabular}{|c|c|c|c|c|}
\hline Selected works & Description and main contributions & $\begin{array}{l}\text { Compared against } \\
\text { health-care } \\
\text { professionals }\end{array}$ & $\begin{array}{l}\text { Is the } \\
\text { dataset } \\
\text { public? }\end{array}$ & Year \\
\hline DeVries et al. (56) & $\begin{array}{l}\text { CNN trained on a public dataset for a skin lesion classification challenge. CNN } \\
\text { uses two different scales of input images to obtain satisfactory results }\end{array}$ & No & Yes & 2017 \\
\hline Mahbod et al. (57) & $\begin{array}{l}\text { Automated computational method for skin lesion classification based on three } \\
\text { CNNs that act as visual feature extractors. Features are combined with SVM } \\
\text { whose outputs are merged to generate a final result. Training and validation } \\
\text { steps are performed with a public dataset. Satisfactory performance on } 150 \\
\text { validation images of the public dataset }\end{array}$ & No & Yes & 2017 \\
\hline Harangi et al. (58) & $\begin{array}{l}\text { Classification method for } 3 \text { different skin lesions based on an ensemble of } \\
4 \text { CNNs trained on public datasets. Method fuses outputs of classification } \\
\text { layers of the four CNNs. Final classification is obtained by a weighted output } \\
\text { of each of the CNNs. Results are satisfactory }\end{array}$ & No & Yes & 2018 \\
\hline \multirow[t]{2}{*}{ Menegola et al. (59) } & $\begin{array}{l}\text { Ensemble of CNNs combining outputs with a SVM final decision. Several public } \\
\text { datasets were combined into one to train the CNNs }\end{array}$ & No & Yes & 2017 \\
\hline & $\begin{array}{l}\text { Results are satisfactory for two binary classification problems, melanoma vs. all } \\
\text { and keratosis vs. all }\end{array}$ & & & \\
\hline \multirow[t]{2}{*}{ Liu et al. (60) } & $\begin{array}{l}\text { Deep learning system (DLS) identifies } 26 \text { common skin conditions in adults } \\
\text { referred for teledermatology consultation (60). DLS provides full differential } \\
\text { diagnosis across those } 26 \text { conditions }\end{array}$ & Yes & No & 2020 \\
\hline & $\begin{array}{l}\text { System diagnostic accuracy was compared against clinicians. Its performance } \\
\text { is comparable to dermatologists and more accurate than general practitioners }\end{array}$ & & & \\
\hline
\end{tabular}

SVM, Support Vector Machines; CNN, convolutional neural network.

\section{State of the art}

There is a variety of recent publications that utilize the tools and models of DL to address the problem of skin lesion classification, the use of these techniques has achieved a much better performance in terms of metrics such as accuracy, specificity and sensitivity in comparison with the results obtained with classical ML techniques. Some examples of them can be found in (53-59).

One of the most relevant articles about the applications and the potential that DL can offer in the field of dermatology was presented by Esteva et al. (54). The performance of this network was tested against 21 certified dermatologists in two critical cases: carcinomas $v s$. seborrheic keratosis and melanomas vs. nevus. Research concludes that the convolutional neuronal network achieves a superior performance than that reached by experts in dermatology. The use of the system in mobile devices to facilitate an early diagnosis is proposed.

Table 4 presents a review of some of the relevant works mentioned above. The last two columns respond to two important aspects: if the performance of the proposed 


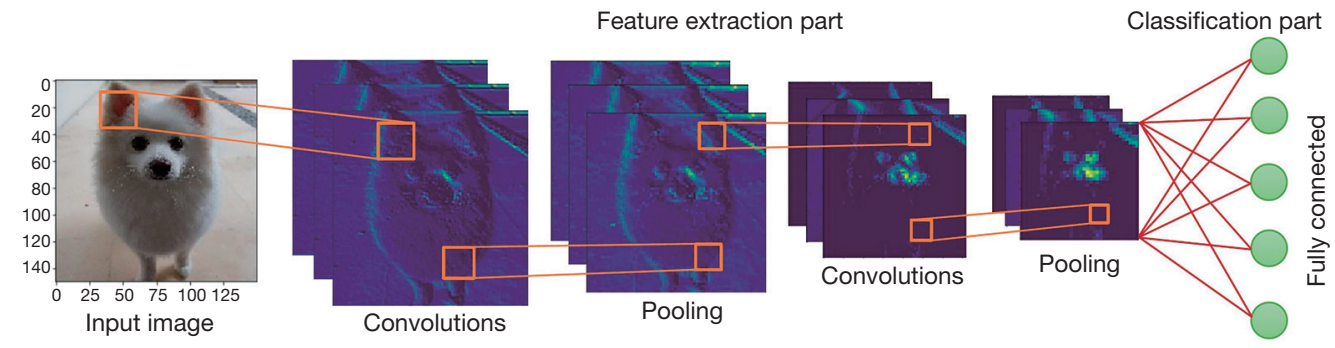

Figure 6 Simplified architecture of a Convolutional Neural Network.

method was compared with that of health professionals and if the dataset used to train the neural network models is publicly available, by "public" we mean whether the images belong to a well-known dataset or whether the authors collected their data from different public and private sources and ultimately made the final dataset public. This last aspect is important as it is one of the factors that allows the results to be reproduced and compared with other techniques.

\section{CNNs for dermatology}

In Figure 6, we show the typical architecture of a CNN. CNNs are composed of several convolution and pooling layers that act as automatic feature extractors for images, the fully connected network receives the relevant features as input and acts as a classifier. $\mathrm{CNNs}$ can be trained to perform a widely different variety of tasks, they provide a way to train superhuman image classifiers (61).

State of the art deep CNNs (YOLOv3) (62) have been trained in conjunction with image processing techniques (segmentation algorithms) to deploy a skin lesion detection system that learns to detect (localize and classify) three types of skin lesions: melanoma, nevus, and seborrheic keratosis. The more general case, detecting benign from malignant skin lesions has also been addressed. Some of the visual results are shown in Figure 7. The DL-based detection system can predict a confidence value (probability) for the nature of the skin lesion under analysis at three different scales.

Results of Figure 7 were verified by a dermatologist (to assure that the skin lesion in the image corresponded to the label attached to it). They belong to a test partition which was never seen by the deep neural network. The test partition was conformed from publicly available images from different healthcare websites.

For training the neural network, images from the
International Skin Imaging Collaboration archive (ISIC archive) were used. The ISIC archive is a high quality dermoscopic image database, collected from international leading clinical centers and acquired from a variety of devices. At the time of preparing this work, the ISIC archive dataset consisted of 19,330 benign lesions and 2,286 malignant lesions. It is important to remark that DL models learn the most when they are trained with the largest possible number of images.

\section{Future trends in AI for dermatology}

One of the most recent works on the skin lesion classification problem was presented by Google Health in collaboration with other institutions like the University of California-San Francisco, the Massachusetts Institute of Technology and the Medical University of Graz. They developed a Deep Learning System (DLS) capable of delivering a differential diagnosis of the most common skin conditions seen in primary care. Unlike previous approaches, this system can exploit the information present in the images as well as in the metadata, achieving an accuracy across 26 skin conditions, on par with U.S. boardcertified dermatologists (60).

DL-based systems can be of great use for dermatologists, they can be put to work as a desktop automatic diagnostic app or can be embedded into a smartphone attached to a mobile dermatoscope. We seek to deploy, in the near future, a real-time DL solution in a smartphone app that could be used by general practitioners and the general public. As stated by Liu et al. (60), a solution of this kind could be useful in regions where there is a lack of medical specialists in local clinics. This technology has the potential to have an impact on public health as it can increase the capabilities of general practitioners and help in the decision-making process. 

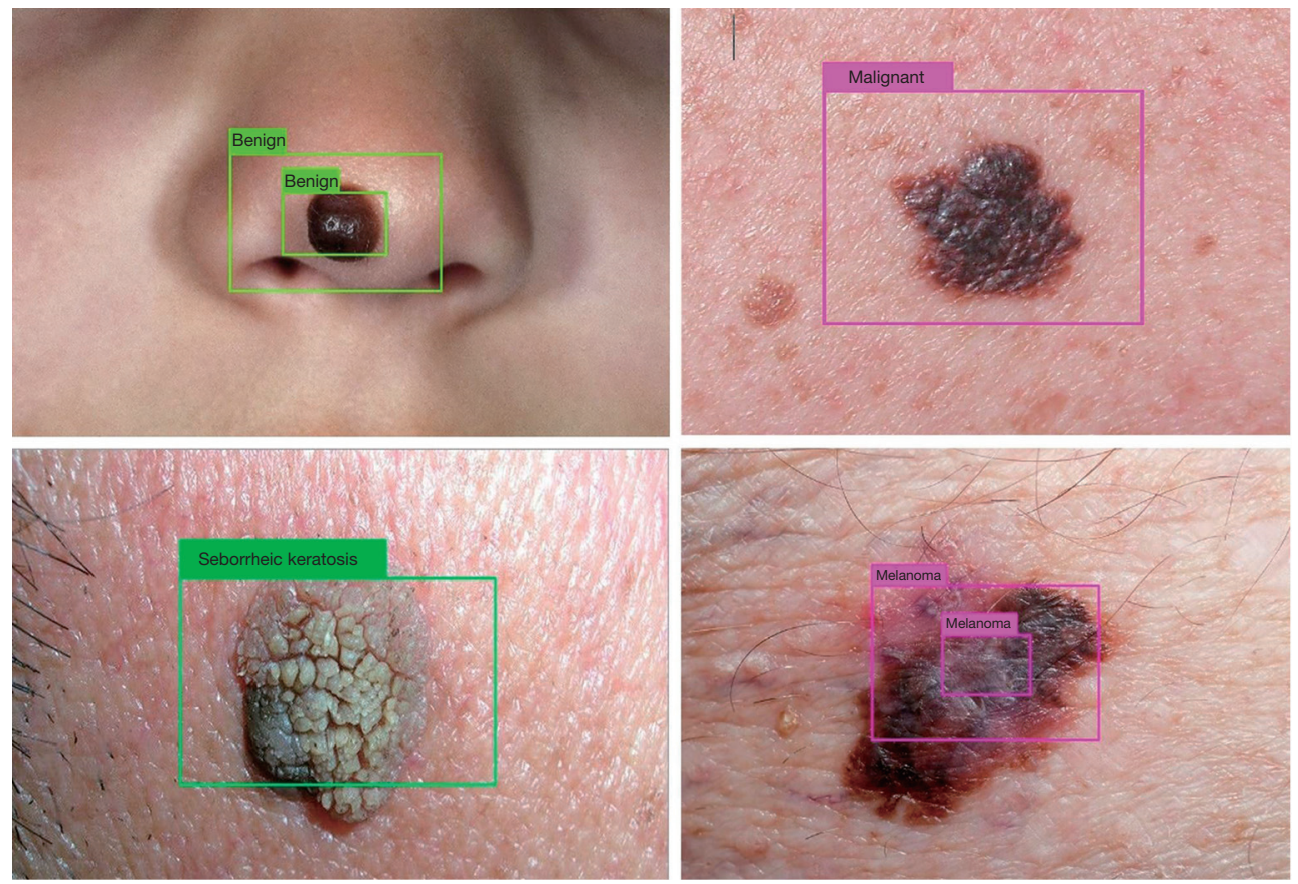

Figure 7 Processed test images by the trained deep neural network. Nevi correctly detected as benign and melanoma correctly detected as malignant (upper row). Seborrheic keratosis (benign lesion) and melanoma correctly detected (lower row).

\section{Microscopy image analysis for cancer}

Microscopy image acquisition modalities use the light spectrum of electromagnetic radiation and its interaction with internal tissues to evaluate molecular changes. In contrast to other acquisition techniques, microscopy takes advantage of various colors of light to visualize and measure several tissue features with improved contrast and resolution. These features also have potential applications in diagnosis, grading, identification of tissue substructures, prognostication and mutation prediction (63). One of the advantages of microscopy is high spatial resolution. The typical resolution is around hundreds of nanometers, useful for monitoring subcellular features. Superresolution imaging methods can further improve the resolution by one or two orders of magnitude (64). Microscopy takes advantage of a variety of contrast mechanisms, where the most popular stain is hematoxylin and eosin (H\&E).

The pathologists have been beneficiated directly by these applications because they could propose tailored therapies based on individual profiles and spend more time focusing on more complicated cases that are not as quickly diagnosable. Even though the number of digital images generated for diagnostic and therapeutic purposes is increasing rapidly (65), extensive experimental comparisons and stateof-the-art surveys are difficult tasks because most data are not public (66) and because there are substantial differences in acquisition protocols, tissue preparation and stain reactivity (67).

In the field of CAD based on microscopy image assessment, an objective quantitative approach is required to increase reproducibility and predictive accuracy. Microscopy provides relevant information about the tissue since the structure is preserved in the preparation process, and it is considered the diagnosis gold standard for many diseases, including many types of cancer (68).

\section{ML for cancer classification}

In this section, we focus on two types of cancer: hematologic malignancies (HMs) and colorectal. The main idea is the classification of microscopy image databases that include different types of malignancies (hematologic) and tissues (colorectal). The whole classification process involves $\mathrm{CV}$ and $\mathrm{ML}$ algorithms, and the process consists of two significant steps: description and classification. The description of these databases is centered on textural information from the images to compute feature vectors 


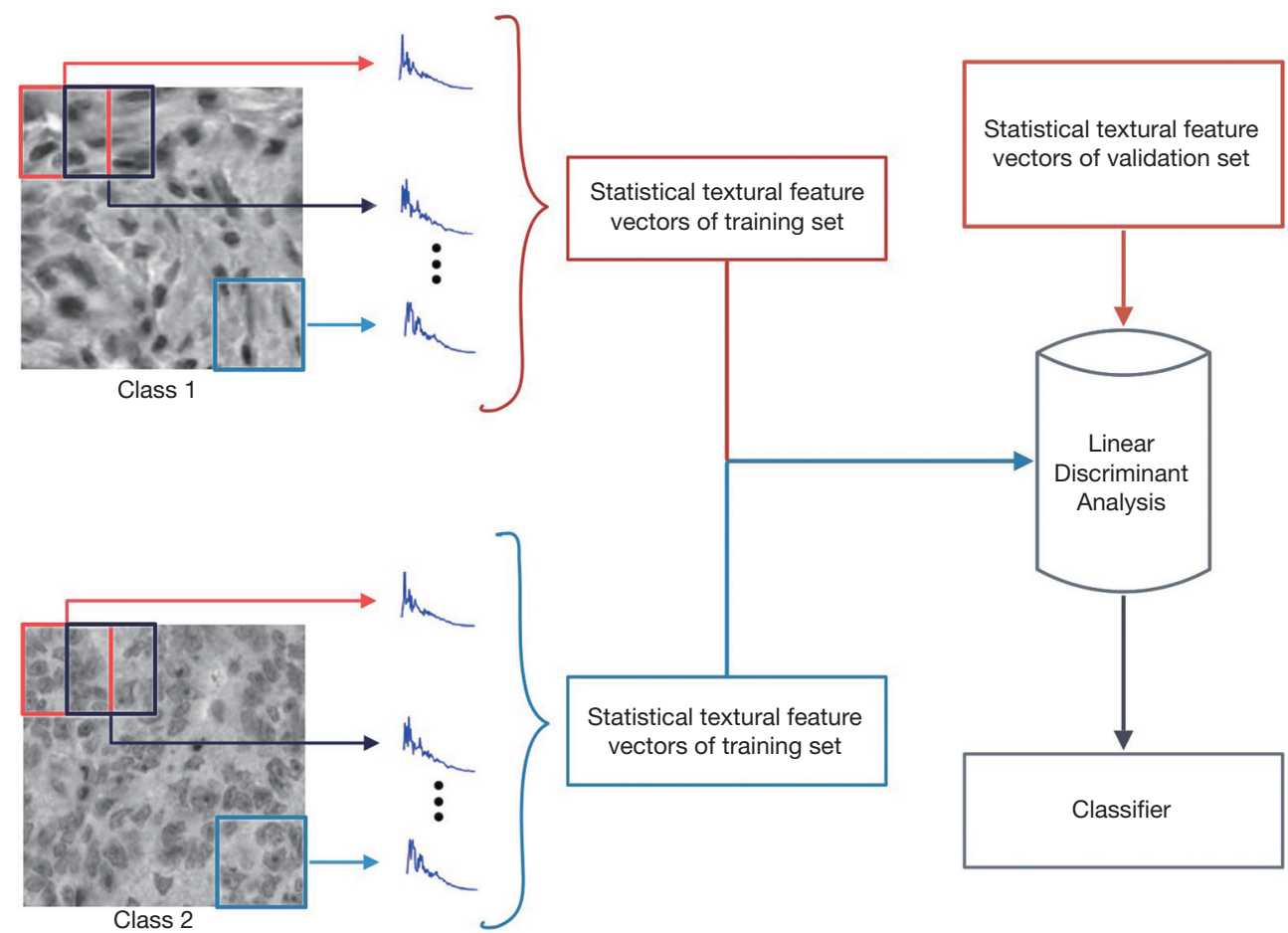

Figure 8 General scheme of classification based on discrete orthogonal moments. After computing local DOMs using sliding windows, the statistical textural feature vectors are computed and classified.

(CV). Then, feature vectors are generated from textural information to be processed by the classifier; in other words, a machine-learning algorithm.

HMs are a collection of diseases that includes leukemia and lymphomas (69). Three types of malignancies will be addressed in this study: Chronic lymphocytic leukemia (CLL), follicular lymphoma (FL), and mantle cell lymphoma (MCL). The IICBU2008-lymphoma dataset (70) includes the three mentioned malignancies, and many automatic methods have been proposed to classify this dataset.

According to GLOBOCAN 2018 data, colorectal cancer (CRC) is the third most deathly and the fourth common type of cancer worldwide, with more than 1.8 million new cases registered in 2018 (71). The Epistroma database (72) is a collection of epithelial and stroma tissue samples taken from patients with histologically verified CRC at the Helsinki University Central Hospital, Helsinki, Finland, from 1989 to 1998.

A classification method based on textural features applied to both colorectal and HMs is based on discrete orthogonal moments (DOMs). The orthogonal moments are computed as the projection of the images on an orthogonal polynomial basis. One way of interpreting the projection is as the correlation measure between the image and the polynomial basis (73). The best known discrete polynomial bases (74), with a vast number of applications in a variety of research areas, are Tchebichef, Krawtchouk, Hahn, and Dual Hahn.

González et al. (75), based on previous papers $(76,77)$, introduced and validated Shmaliy orthogonal moments as a statistical textural descriptor, and its performance is compared to the DOMs. This statistical textural descriptor is based on overlapping square windows and assure numerical stability. They also proposed a classification method with the most popular ML algorithms: SVM, k-NN rule, NB rule, and Random Forest. The general scheme of classification is depicted in Figure 8. This proposal and its detailed description have been published previously for CRC $(78)$ and HMs $(73,75)$ with state-of-art results at the time of publication. Recently, with the spread of neural networks, some papers $(79,80)$ have worked on these databases using CNNs, e.g., Kather et al. (81) compared classical textural descriptors with the most known CNNs architectures applied to different microscopy image datasets. Table 5 summarizes a list of relevant classification methods with different algorithms applied to microscopy 
Table 5 Microscopy image classification. List of representative classification methods on Lymphoma and Epistroma databases

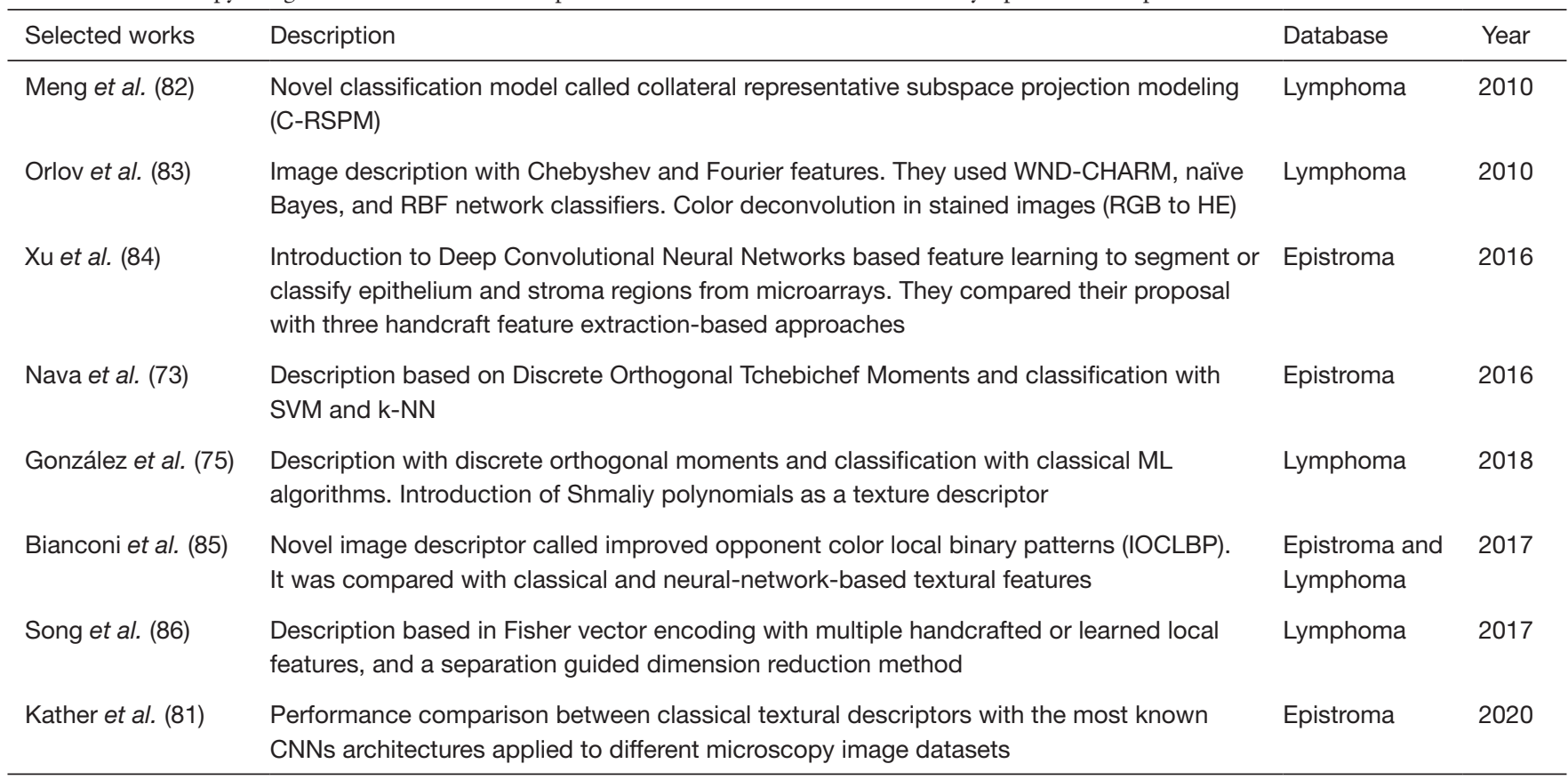

SVM, Support Vector Machines; CNN, convolutional neural network; ML, machine learning.

image datasets, ordered chronologically.

DOMs, in conjunction with ML algorithms, have proven to be particularly useful methods for description and classification of microscopy images. From a future perspective, polynomial bases can be used as modulation filters in addition to CNNs, as Luan et al. (87) show in their paper.

As the area of microscopy imaging continues to develop, both in acquisition techniques and the application of new contrast mechanisms, the processing of these images will also grow. For example, through the cited cases, color processing (i.e., different color spaces and color deconvolution) in microscopy images is recurrent to obtain better classification results among tissues of interest. That improvement depends directly on the acquisition and the contrast mechanism. On the other hand, the more public databases there are, the better profitability from the latest DL classifiers, because more information, means better performance classifiers.

\section{Neurodegenerative diseases and brain structure segmentation}

In recent years, automatic detection of different brain structures has become a challenging task for scientists since the nature of image acquisition often yields issues as noise, blur or intensity inhomogeneities which depend on the specific medical image modality, affecting the correct brain localization and visualization process. The most popular image modalities used for brain analysis are positron emission tomography (PET), single photon emission computed tomography (SPECT) and MRI, the latter becoming a standard tool due to the good quality contrast of brain tissues in a non-invasive way and has proven very efficient for diagnosis, evaluation and monitoring.

Since the process of manual delineation of brain structures is exhausting and time consuming for experts, the need of automated segmentation is urgent, not only to improve accuracy but also to reduce inter and intra observer variability. For instance, the identification of subcortical brain structures, hippocampus, hypothalamus, amygdala, among others, is a challenging task due to their size and shape. Some of these structures possess a special interest for its association with neurodegenerative disorders $(88,89)$.

This section reviews some representative semi-automatic methods based on $\mathrm{CV}$ and AI reported in the literature that tackle brain segmentation problems in medicine and its application nowadays. 


\section{Whole brain segmentation}

Neurodegenerative disorders are usually related to brain alterations such as structure, shape and volume changes. Whole brain segmentation helps providing qualitative and quantitative comparison with other smaller brain structures. FreeSurfer is a software developed by Fischl et al. (90) which provides a set of algorithms to segment the whole brain from T1-weighted images. A particular approach based on Markov random fields is Local Cooperative Unified Segmentation (LOCUS) which was originally designed by Scherrer et al. (91) and includes subcortical segmentation in phantoms and real $3 \mathrm{~T}$ brain scans. Other approaches extract relevant information such as high energy regions and oriented structures to ease the identification of white matter, gray matter and cerebrospinal fluid as stated in (92).

\section{The role of hippocampus in dementia}

Different types of neurodegenerative diseases are originated in different areas of the brain. For instance, hippocampus plays a key role in the prediction of diseases such as Alzheimer, schizophrenia and bipolar disorder, among others. Jorge Cardoso et al. (93) presented a technique for Alzheimer early detection named Similarity and Truth Estimation for Propagated Segmentation (STEPS). It segments the hippocampus with practical applications to Alzheimer's Disease Neuroimaging Initiative (ADNI). ADNI stores a large data collection that attempts to keep record of the progression of Alzheimer disease, including MRI and PET images, blood biomarkers, genetic and cognitive tests. In order to reduce the number of atlases (labeled images) needed to guide the segmentation of the whole hippocampus, Multiple Automatically Generated Templates (MAGeT) is a method proposed by Pipitone et al. (94) to generate templates constructed via label propagation, this method achieves good performance compared to classic multi-atlas methods with a reduced training data.

Adapting segmentation methods to analyze shape and intensity information leads to a robust scheme to deal with common image degradations. An automatic segmentation of the caudate nuclei was constructed by using a probabilistic framework to estimate the relation between shape and pixel intensities. This leads to an important improvement for segmenting subcortical brain structures as proposed by Patenaude et al. (95) and originally applied on evaluating Alzheimer's disease by the software FIRST. In order to improve the segmentation results in the accurate extraction of hippocampus from brain MR images, Gao et al. (96) exploit the capability of atlas-based methods which serves as the initialization for a further implementation in a semiautomatic model. Table 6 summarizes some of the more relevant segmentation methods in this area.

\section{Subcortical structures and brainstem linked to movement disorders}

Electric stimulation of the functional brain regions has been suggested in the literature as a surgical treatment for some movement-related disorders (101). Surgical planning of these procedures implies segmenting automatically the appropriate region with high accuracy. For instance, brainstem is relevant to describe disorders as Parkinson or progressive supranuclear palsy. Brainstem includes structures like midbrain, pons, medulla oblongata and substantia nigra. Several diseases are directly related to atrophy, reduced nigra volume and structure deformations. Particularly, Li et al. (97) explored the literature to provide a conclusive relation of substantia nigra concentration in Parkinson's patients. Complementary works have developed methods to increase accuracy in midbrain segmentation by including texture descriptors and performing local analysis as proposed by Olveres et al. (98) whose results are displayed in Figure 9.

Among the subcortical structures, the basal ganglia are located in the center of the brain and consist of several different groups of neurons. Neuronal death means that these groups of neurons can no longer work properly, causing stumbling and shaking. In order to improve classic segmentation techniques with subcortical applications, DLbased methods have emerged as successful alternatives. As shown by Milletari et al. (99), DL results may vary depending on the architecture used. Authors showed a segmentation comparative in MRI and transcranial ultrasound volumes of the basal ganglia and midbrain. The incorporation of probabilistic atlases as spatial features allows neural networks to segment the most difficult areas of subcortical brain structures, Kushibar et al. (100).

\section{Respiratory diseases}

\section{COPD}

There are several important respiratory diseases, such as COPD, asthma, occupational lung diseases and pulmonary 
Table 6 Brain structure segmentation. A summary of representative methods for brain segmentation

\begin{tabular}{|c|c|c|c|}
\hline Selected works & Description & Type of images & Structure(s) \\
\hline Scherrer et al. (91) & $\begin{array}{l}\text { LOCUS (Local Cooperative Unified Segmentation) based on } \\
\text { Markov Random Fields }\end{array}$ & T3 MRI & Subcortical \\
\hline Carbajal-Degante et al. (92) & Texture-based approach with Hermite coefficients and level sets & $\mathrm{MRI}$ & Brain tissue \\
\hline Pipitone et al. (94) & MAGeT (Multiple Automatically Generated Templates) & Templates & Hippocampus \\
\hline Patenaude et al. (95) & Alzheimer's evaluation by the software called FIRST & T1-weighted MRI & Subcortical \\
\hline Gao et al. (96) & Semiautomatic atlas-based & $\mathrm{MRI}$ & Hippocampus \\
\hline Li et al. (97) & Substantia nigra concentration in Parkinson's patients & Ultrasound, TCS & Brainstem \\
\hline Kushibar et al. (100) & Spatial and deep convolutional features & MRI & Subcortical \\
\hline
\end{tabular}

MRI, magnetic resonance imaging; PET, positron emission tomography; TCS, transcranial sonography; CNN, convolutional neural network.
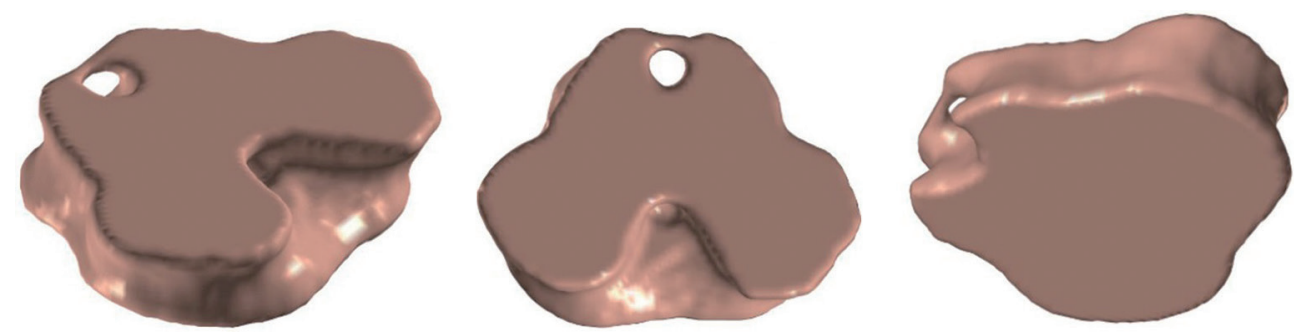

Figure 9 Segmentation results of midbrain in MRI by Olveres et al. (99).

hypertension. According to WHO (102), COPD is a progressive lung disease that causes breathlessness (initially with exertion) and predisposes to exacerbations and serious illness. It is characterized by a persistent reduction of airflow. Globally, it is estimated that it accounts for $5 \%$ of all deaths until 2019. More than $90 \%$ of COPD deaths occur in low and middle-income countries and the primary cause of COPD is exposure to tobacco smoke (either active smoking or secondhand smoke). Traditionally diagnosis of COPD is done by spirometry; however, this method might underdiagnose considerably (103).

Recently, several papers like the Bibault et al. and Mekov et al. $(104,105)$, propose digital image modalities and AI using low dose CT images to detect signs of COPD with DL techniques. Even when the disease is not curable, results offer an opportune diagnosis which allows treatment to improve life quality in COPD patients. In order to pay attention to the lungs area, where lesions occur, several segmentation algorithms have been developed and, nowadays, this task can be executed with traditional as well as DL methods $(106,107)$. COPD detection is treated in Rajpurkar et al. (108) and Shi et al. (109) reviews several methods of segmentation for severe COVID-19. These tasks are accomplished using $\mathrm{X}$ radiographies and chest $\mathrm{CT}$ images.

There is an increasing number of studies that detect pneumonia on chest radiographies like the one of Zech et al. (110). CT images have proven to have enough spatial resolution to obtain specific metrics of pneumonia lesions needed to estimate a damage level according to 
Table 7 Respiratory diseases. Selection of relevant works of different methods in respiratory area

\begin{tabular}{|c|c|c|c|}
\hline Selected works & Description & Type of images & Year \\
\hline Mekov et al. (105) & Use artificial intelligence and machine learning to detect signs of COPD & СT & 2020 \\
\hline Afzali et al. (106) & Use of contour-based lung shape analysis for tuberculosis detection & Chest Xray & 2020 \\
\hline Shi et al. (109) & $\begin{array}{l}\text { Review of Artificial Intelligence Techniques in Imaging Data Acquisition, } \\
\text { Segmentation and Diagnosis for COVID-19 }\end{array}$ & CT and X-ray & 2021 \\
\hline Castillo-Saldana et al. (111) & $\begin{array}{l}\text { Obtain specific metrics of pneumonia lesions to give a damage degree for chronic } \\
\text { obstructive pulmonary disease and fibrotic interstitial lung disease }\end{array}$ & CT & 2020 \\
\hline Li et al. (113) & $\begin{array}{l}\text { Use of artificial Intelligence to distinguish between COVID-19 from Community } \\
\text { Acquired Pneumonia on chest CT }\end{array}$ & Chest CT image & 2020 \\
\hline
\end{tabular}

CT, computer tomography; COPD, chronic obstructive pulmonary disease.

Castillo-Saldana et al. (111). They provide objective densitybased and texture-based evaluation of the lungs. New methods of measuring airways have recently been developed that complete a prognosis and guide clinical decisions.

On the other hand, studies demonstrate that COPD may also increase the risk of contracting severe COVID-19 (112), another important respiratory disease to analyze. Table 7 presents some of the relevant works mentioned in this section.

\section{COVID-19}

The impact of the COVID-19 worldwide crisis has triggered efforts in research communities and interdisciplinary groups to collaborate and search for solutions to mitigate this disease effects. CT imaging and X-ray studies have been helpful in the prognosis for patients since they allow to detect characteristic lesions such as ground-glass opacity, consolidation, pleural effusion, bilateral involvement, peripheral and diffuse distribution (113). However, with the pandemic generated by COVID-19, the lack of radiological expertise and the need for precise interpretation chest medical images has become evident. AI solutions aim at helping the clinical area to face this worldwide crisis.

Image-analysis research groups have focused on building computer aided diagnosis systems to detect COVID-19 from the automatic analysis of lung chest radiography and CT images. These AI systems provide a probability of existence of COVID-19 as well as detect, segment and quantify lesions in the lungs $(109,113)$. Figure 10 shows the results delivered by one of such systems that are already available online.

\section{Gastrointestinal and liver diseases}

Another important area in which $\mathrm{AI}$ and $\mathrm{CV}$ have had impact is gastroenterology and hepatology. Le Berre et al. (114) made a ten-year compilation of contributions ranging from $M L$ to DL technologies. In the endoscopic area $\mathrm{AI}$ has been used in lesions analysis, cancer detection and inflammatory lesions analysis. They present 53 studies, most of them related to endoscopy, that detect or improve diagnosis of intestinal lesions, polyp's cancer, as well as premalignant and malignant analysis of the upper gastrointestinal tract, and/or the entire digestive tract. With the assistance of AI algorithms, performances are above $80 \%$. Kudo et al. (115) identified neoplasms through endoscopic images with high accuracy and low interobserver variation. They identified colon lesions with $96.9 \%$ sensitivity, 100\% specificity and 98\% accuracy. Diagnostic accuracy of their EndoBRAIN system was calculated on a multi-center study using 69,142 endocytoscopic images.

AI has also been used in the study of inflammatory bowel diseases, ulcers. celiac disease, lymphangiectasia and hookworm (114), as well as in identifying the risk of possible bleeding of inflammatory bowel with an accuracy above $90 \%$.

As seen above, most contributions of AI in gastroenterology have been applied on endoscopy images. In the case of liver diseases, AI has been found useful in other image modalities as described below. Table 8 presents some of the relevant works in the gastroenterology area. 


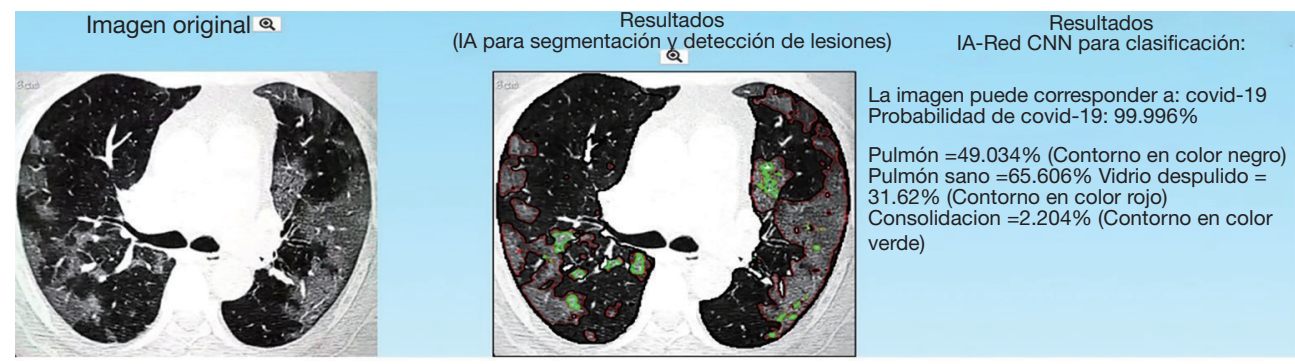

Figure 10 AI-based online tool that predicts COVID-19 and segments (green and red contours) lung lesions associated to the disease (https://www.imagensalud.unam.mx).

Table 8 Gastrointestinal and liver diseases. Selection of relevant works of different methods in gastrointestinal area

\begin{tabular}{|c|c|c|c|}
\hline Selected works & Description & Type of images & Year \\
\hline Kudo et al. (115) & $\begin{array}{l}\text { Identification of neoplasms through endoscopic images with high accuracy and low } \\
\text { interobserver variation }\end{array}$ & Endoscopy & 2020 \\
\hline Liu et al. (117) & CNN method to detect liver cirrhosis with classification accuracy of $86.9 \%$ & US & 2017 \\
\hline Biswas et al. (118) & $\begin{array}{l}\text { Deep learning technique for detection of hypoechoic fatty liver diseases and stratification } \\
\text { of normal and abnormal (fatty) ultrasound liver images }\end{array}$ & US & 2018 \\
\hline Yasaka et al. (119) & $\begin{array}{l}\text { CNN lesions classification method with an average accuracy of } 84 \% \text { for several liver } \\
\text { lesions, malignant tumors, other mass-like lesions, and cysts }\end{array}$ & CT & 2018 \\
\hline
\end{tabular}

US, ultrasound; CT, computed tomography; CNN, convolutional neural network.

\section{Liver diseases}

Nonalcoholic fatty liver disease (NAFLD) comprises a broad clinical spectrum which ranges from simple hepatic steatosis to more advanced stages such as non-alcoholic steatohepatitis (NASH), liver cirrhosis, and in certain cases it can lead to hepatocellular carcinoma (HCC) $(121,122)$. Currently, it is considered the most prevalent chronic liver disease worldwide.

Predictions show a discouraging picture which brings greater costs associated with health, and a worse prognosis for patients. In this context, it is very important to identify patients who are at a higher risk of developing chronic liver disease in order to prevent cirrhosis progression or development of HCC $(123,124)$.

AI has been used to assess patients with this disease, 13 studies used data from electronic medical records and/or biologic features to build the algorithms and three studies used data from elastography. These models identified their target factor with approximately $80 \%$ accuracy (114).

Several authors have applied AI to assist liver disease diagnosis, such as focal liver lesion detection, segmentation and evaluation, as well as diffuse liver staging. Most techniques use CT, MRI or Ultrasound images and the reported results are very promising.

In the case of tumor detection methods based on $\mathrm{CNN}$ with CT images have shown to increase the precision rate to $86 \%$ compared with the $72 \%$ of human performance (116). In the case of ultrasound Liu et al. (117) developed a CNN method that can detect liver cirrhosis with a classification accuracy of $86.9 \%$. In the case of fat liver disease and stratification Biswas et al. (118) applied a DL technique for detection of hypoechoic fatty liver diseases and stratification of normal and abnormal (fatty) ultrasound liver images. They created a ML application for tissue characterization 
and risk assessment, composed of three different algorithms, namely SVM, extreme learning machines and a DL $\mathrm{CNN}$ model. The ultrasound images of 63 patients were annotated as normal and abnormal depending upon the biopsy laboratory tests. The report shows an astonishing accuracy of $100 \%$ with the DL CNN system.

Recent developments have been directed to specific problems such as detection of fatty liver disease, tumor detection, metastasis prediction with high performance rates, often above $90 \%$ of accuracy (125).

An advanced method (119) based on a CNN with CT images shows an average accuracy of $84 \%$ in the classification of several liver lesions, i.e., HCC, other malignant tumors, other mass-like lesions, and cysts.

Ibragimov et al. (120) showed a Dice coefficient of $83 \%$ with a CNN working on CT images in the case of portal vein segmentation.

The future of AI in liver medical care lies in the implementation of fully automated clinical tasks to help complex diagnosis. This implies to overcome present day limitations of ML technique such as multiple tasking. Massive efforts to gather annotated image and clinical data are also needed.

\section{Discussion and conclusions}

In this paper we presented several examples where $\mathrm{CV}$ and ML have been successfully used in medical applications in different organs, pathologies, and image modalities. These technologies have the potential to impact on public health as they can increase the capabilities of general practitioners and help in the standardization of the decision-making process, especially in regions where there is a lack of medical specialists in local clinics. Although these advantages have push towards constant update of the performance results across different applications, there exist some challenges that are commonly encountered, giving room for improvement.

Though multiple databases have emerged in the last decade there is still need for more information in the medical field, the need of databases with expert-annotated ground truth that supports the creation and performance of these methods is still an important issue. Benchmark datasets, accessible to the public, can be valuable to compare existing approaches, discover useful strategies and help researchers to build better approaches. The lack of up-to-date benchmark databases is due to several factors, among them, restrictions in clinical environments and lack of medical experts willing to annotate large amounts of data. The latter being a task that is challenging since manual segmentation is a tedious task that is prone to errors due to inter and intra observer variability. Although an incredible effort has been done, including many years of work and large quantities of spending resources, the amount of sufficient and balanced data to evaluate the performance of different techniques applied to the medical field is scarce, compared with the large amount of publicly available datasets in other areas such as ImageNet, COCO dataset, Google's Open Images. There is a clear need of new medical workflows that enables to overcome the abovementioned problems, but it is equally important to foster research on new AI techniques that are less dependent on big data quantities neither so computationally demanding.

Although transfer learning and data augmentation are commonly used to address the issue of small datasets, research on how to improve cross-domain and crossmodal learning and augmentation in the medical field is a most needed task. A promising paradigm in ML is metalearning. This includes a series of methods that aim at using previously learned knowledge to solve related tasks. In medical imaging this opens new possibilities to overcome the problem of limited data sets. For instance, a model that learned to classify anatomical structures in a given modality, e.g., CT, can use that knowledge to classify the same structures in other modalities like MRI or ultrasound. Likewise, a model that learned to segment a given structure, e.g., a cardiac cavity, can use that knowledge to learn to segment other cavities without the need to train a new model from scratch.

Another topic that has recently called for attention in automatic methods for medical imaging is the need to account not only with high accuracy and precision but also with a measure of how certain the response given by an automatic method is. This is the case, for instance, of the Bayesian DL networks that include a probabilistic model that allows to quantify the uncertainty of a decision. An uncertainty measure provides the radiologist with an additional confidence parameter to emit a diagnosis assisted by an automatic method.

Finally, hybrid methods that combine conventional ML techniques with current state-of-the-art DL methods are also promising alternatives that have shown significant improvements over single methods. In addition, current computing capabilities have allowed these techniques to 
become increasingly popular, opening new challenges to the scientific community for developing and implementing fully automated real-time clinical tasks to help complex diagnosis and procedures.

\section{Acknowledgments}

Funding: This work has been sponsored by UNAM PAPIIT grants TA101121 and IV100420, and SECTEI grant 202/2019.

\section{Footnote}

Conflicts of Interest: All authors have completed the ICMJE uniform disclosure form (available at http://dx.doi. org/10.21037/qims-20-1151). The authors have no conflicts of interest to declare.

Ethical Statement: The authors are accountable for all aspects of the work in ensuring that questions related to the accuracy or integrity of any part of the work are appropriately investigated and resolved.

Open Access Statement: This is an Open Access article distributed in accordance with the Creative Commons Attribution-NonCommercial-NoDerivs 4.0 International License (CC BY-NC-ND 4.0), which permits the noncommercial replication and distribution of the article with the strict proviso that no changes or edits are made and the original work is properly cited (including links to both the formal publication through the relevant DOI and the license). See: https://creativecommons.org/licenses/by-nc-nd/4.0/.

\section{References}

1. GBD 2013 Mortality and Causes of Death Collaborators. Global, regional, and national age-sex specific all-cause and cause-specific mortality for 240 causes of death, 19902013: a systematic analysis for the Global Burden of Disease Study 2013. Lancet 2015;385:117-71.

2. Beard JR, Officer A, de Carvalho IA, Sadana R, Pot AM, Michel JP, Lloyd-Sherlock P, Epping-Jordan JE, Peeters GMEEG, Mahanani WR, Thiyagarajan JA, Chatterji $\mathrm{S}$. The World report on ageing and health: a policy framework for healthy ageing. Lancet 2016;387:2145-54.

3. Marengoni A, Angleman S, Melis R, Mangialasche F, Karp A, Garmen A, Meinow B, Fratiglioni L. Aging with multimorbidity: a systematic review of the literature.
Ageing Res Rev 2011;10:430-9.

4. Dagenais GR, Leong DP, Rangarajan S, Lanas F, LopezJaramillo P, Gupta R, Diaz R, Avezum A, Oliveira GBF, Wielgosz A, Parambath SR, Mony P, Alhabib KF, Temizhan A, Ismail N, Chifamba J, Yeates K, Khatib R, Rahman O, Zatonska K, Kazmi K, Wei L, Zhu J, Rosengren A, Vijayakumar K, Kaur M, Mohan V, Yusufali A, Kelishadi R, Teo KK, Joseph P, Yusuf S. Variations in common diseases, hospital admissions, and deaths in middle-aged adults in 21 countries from five continents (PURE): a prospective cohort study. Lancet 2020;395:785-94.

5. World Health Organization. WHO report on cancer: setting priorities, investing wisely and providing care for all. Geneva: World Health Organization, 2020. License: CC BY-NC-SA 3.0 IGO.

6. Wolff JL, Starfield B, Anderson G. Prevalence, expenditures, and complications of multiple chronic conditions in the elderly. Arch Intern Med 2002;162:2269-76.

7. Maresova P, Javanmardi E, Barakovic S, Barakovic Husic J, Tomsone S, Krejcar O, Kuca K. Consequences of chronic diseases and other limitations associated with old age - a scoping review. BMC Public Health 2019;19:1431.

8. Kontos D, Summers RM, Giger M. Special Section Guest Editorial: Radiomics and Deep Learning. J Med Imaging (Bellingham) 2017;4:041301.

9. Erickson BJ, Korfiatis P, Akkus Z, Kline TL. Machine Learning for Medical Imaging. Radiographics 2017;37:505-15.

10. Patel VL, Shortliffe EH, Stefanelli M, Szolovits P, Berthold MR, Bellazzi R, Abu-Hanna A. The coming of age of artificial intelligence in medicine. Artif Intell Med 2009;46:5-17.

11. Moor J. The Dartmouth College Artificial Intelligence Conference: The Next Fifty Years. AI Magazine 2006;27:87.

12. Kononenko I. Machine learning for medical diagnosis: history, state of the art and perspective. Artif Intell Med 2001;23:89-109.

13. Krizhevsky A, Sutskever I, Hinton GE. ImageNet Classification with Deep Convolutional Neural Networks. In: Pereira F, Burges CJC, Bottou L, Weinberger KQ, editors. NeurIPS Proceedings (2018). Curran Associates, Inc., 2012:1097-105

14. Shen D, Wu G, Suk HI. Deep Learning in Medical Image Analysis. Annu Rev Biomed Eng 2017;19:221-48.

15. The ISIC 2020 Challenge Dataset. 2020 Jun. [accessed 29 Jun, 2020]. Available online: https://challenge2020.isicarchive.com/ 
16. World Health Organization. Cardiovascular diseases (CVD) 2019. Available online: https://www.who.int/ health-topics/cardiovascular-diseases/. [Online; accessed: May 11th 2020].

17. Ventura-Clapier R. Encyclopedia of Exercise Medicine in Health and Disease. Springer Berlin Heidelberg, 2012.

18. Chen C, Qin C, Qiu H, Tarroni G, Duan J, Bai W, Rueckert D. Deep Learning for Cardiac Image Segmentation: A Review. Front Cardiovasc Med 2020;7:25.

19. Petitjean C, Dacher JN. A review of segmentation methods in short axis cardiac MR images. Med Image Anal 2011;15:169-84.

20. Rizzo S, Botta F, Raimondi S, Origgi D, Fanciullo C, Morganti AG, Bellomi M. Radiomics: the facts and the challenges of image analysis. Eur Radiol Exp 2018;2:36.

21. Sunnybrook Cardiac Data 2009. Cardiac MR Left Ventricle Segmentation Challenge. Available online: http://www.cardiacatlas.org/studies/sunnybrook-cardiacdata/. [Online; accessed: January 26th 2020].

22. CAMUS Database. Available online: https://www.creatis. insa-lyon.fr/Challenge/camus. 2019. [Online; accessed: December 1st 2019].

23. Carbajal-Degante E, Avendaño S, Ledesma L, Olveres J, Escalante-Ramírez B. Active contours for multiregion segmentation with a convolutional neural network initialization. SPIE Photonics Europe Conference, 2020:36-44.

24. Avendaño S, Olveres J, Escalante-Ramírez B. Segmentación de Imágenes Médicas mediante UNet. In: Reunión Internacional de Inteligencia Artificial y sus Aplicaciones RIIAA 2.0, Aug 2019

25. Zhang J, Gajjala S, Agrawal P, Tison GH, Hallock LA, Beussink-Nelson L, Lassen MH, Fan E, Aras MA, Jordan C, Fleischmann KE, Melisko M, Qasim A, Shah SJ, Bajcsy R, Deo RC. Fully Automated Echocardiogram Interpretation in Clinical Practice. Circulation 2018;138:1623-35.

26. Mira C, Moya-Albor E, Escalante-Ramírez B, Olveres J, Brieva J, Venegas E. 3D Hermite Transform Optical Flow Estimation in Left Ventricle CT Sequences. Sensors (Basel) 2020;20:595.

27. WHO. WHO position paper on mammography screening. 2014. Available online: https://www.who.int/cancer/ publications/mammography_screening/en/

28. Devolli-Disha E, Manxhuka-Kërliu S, Ymeri H, Kutllovci A. Comparative accuracy of mammography and ultrasound in women with breast symptoms according to age and breast density. Bosn J Basic Med Sci 2009;9:131-6.
29. Stavros AT, Thickman D, Rapp CL, Dennis MA, Parker SH, Sisney GA. Solid breast nodules: use of sonography to distinguish between benign and malignant lesions. Radiology 1995;196:123-34.

30. Chen DR, Chang RF, Wu WJ, Moon WK, Wu WL. 3-D breast ultrasound segmentation using active contour model. Ultrasound Med Biol 2003;29:1017-26.

31. Moon WK, Lo CM, Chen RT, Shen YW, Chang JM, Huang CS, Chen JH, Hsu WW, Chang RF. Tumor detection in automated breast ultrasound images using quantitative tissue clustering. Med Phys 2014;41:042901.

32. Madabhushi A, Metaxas DN. Combining low-, highlevel and empirical domain knowledge for automated segmentation of ultrasonic breast lesions. IEEE Trans Med Imaging 2003;22:155-69.

33. Abd-Elmoniem KZ, Youssef AB, Kadah YM. Realtime speckle reduction and coherence enhancement in ultrasound imaging via nonlinear anisotropic diffusion. IEEE Trans Biomed Eng 2002;49:997-1014.

34. Bader W, Böhmer S, van Leeuwen P, Hackmann J, Westhof G, Hatzmann W. Does texture analysis improve breast ultrasound precision? Ultrasound Obstet Gynecol 2000;15:311-6.

35. Liu B, Cheng HD, Huang J, Tian J, Tang X, Liu J. Fully automatic and segmentation-robust classification of breast tumors based on local texture analysis of ultrasound images. Pattern Recognit 2010;43:280-98.

36. Chen WM, Chang RF, Kuo SJ, Chang CS, Moon WK, Chen ST, Chen DR. 3-D ultrasound texture classification using run difference matrix. Ultrasound Med Biol 2005;31:763-70.

37. Xian M, Zhang Y, Cheng HD, Xu F, Zhang B, Ding J. Automatic breast ultrasound image segmentation: A survey. Pattern Recognit 2018;79:340-55.

38. Torres F, Escalante-Ramirez B, Olveres J, Yen PL. Lesion Detection in Breast Ultrasound Images Using a Machine Learning Approach and Genetic Optimization. Lecture Notes in Computer Science, 2019:289-301.

39. Yap MH, Edirisinghe EA, Bez HE. A novel algorithm for initial lesion detection in ultrasound breast images. J Appl Clin Med Phys 2008;9:2741.

40. Mainiero MB, Goldkamp A, Lazarus E, Livingston L, Koelliker SL, Schepps B, Mayo-Smith WW. Characterization of breast masses with sonography: can biopsy of some solid masses be deferred? J Ultrasound Med 2005;24:161-7.

41. Huang SF, Chen YC, Woo KM. Neural network analysis applied to tumor segmentation on $3 \mathrm{D}$ breast ultrasound 
images. 2008 Proc IEEE Int Symp Biomed Imaging, 2008:1303-6.

42. Cao Z, Duan L, Yang G, Yue T, Chen Q. An experimental study on breast lesion detection and classification from ultrasound images using deep learning architectures. BMC Med Imaging 2019;19:51.

43. Chiao JY, Chen KY, Liao KY, Hsieh PH, Zhang G, Huang TC. Detection and classification the breast tumors using mask R-CNN on sonograms. Medicine (Baltimore) 2019;98:e15200.

44. Huang Q, Luo Y, Zhang Q. Breast ultrasound image segmentation: a survey. Int J Comput Assist Radiol Surg 2017;12:493-507.

45. Fenster A, Parraga G, Bax J. Three-dimensional ultrasound scanning. Interface Focus 2011;1:503-19.

46. Al-Dhabyani W, Gomaa M, Khaled H, Fahmy A. Dataset of breast ultrasound images. Data Brief 2019;28:104863.

47. Kanitakis J. Anatomy, histology and immunohistochemistry of normal human skin. Eur J Dermatol 2002;12:390-401.

48. American Cancer Society. Cancer Facts \& Figures 2019. Available online: https://www.cancer.org/research/cancerfacts-statistics/all-cancer-facts-figures/cancer-factsfigures-2019.html [Online; accessed: December 1st 2019].

49. Schadendorf D, Hauschild A. Melanoma in 2013: Melanoma-the run of success continues. Nat Rev Clin Oncol 2014;11:75.

50. Camacho LCP, Gerson CR, Góngora JMÁ, Villalobos PA, Blanco VYC, López RO. Actualidades para el tratamiento del melanoma metastásico, estado del arte. Anales Médicos de la Asociación Médica del Centro Médico ABC 2017;62:196-207.

51. Orendain-Koch N, Ramos-Álvarez MP, Ruiz-Leal AB, Sánchez-Dueñas LE, Crocker-Sandoval AB, SánchezTenorio T, Izquierdo-Álvarez J. Melanoma en la práctica privada en México: un diagnóstico oportuno. Dermatol Rev Mex 2015;59:89-97.

52. Johr RH. Dermoscopy: alternative melanocytic algorithmsthe $\mathrm{ABCD}$ rule of dermatoscopy, menzies scoring method, and 7-point checklist. Clin Dermatol 2002;20:240-7.

53. Codella NCF, Gutman D, Celebi ME, Helba B, Marchetti MA, Dusza SW, Kalloo A, Liopyris K, Mishra N, Kittler H, Halpern A. Skin Lesion Analysis Toward Melanoma Detection: A Challenge at the 2017 International Symposium on Biomedical Imaging (ISBI), Hosted by the International Skin Imaging Collaboration (ISIC). 2018 IEEE 15th International Symposium on Biomedical Imaging (ISBI 2018). IEEE, 2018:168-72.

54. Esteva A, Kuprel B, Novoa RA, Ko J, Swetter SM, Blau
HM, Thrun S. Dermatologist-level classification of skin cancer with deep neural networks. Nature 2017;542:115-8. Erratum in: Nature. 2017 Jun 28;546(7660):686.

55. Fujisawa Y, Otomo Y, Ogata Y, Nakamura Y, Fujita R, Ishitsuka Y, Watanabe R, Okiyama N, Ohara K, Fujimoto M. Deep-learning-based, computer-aided classifier developed with a small dataset of clinical images surpasses board-certified dermatologists in skin tumour diagnosis. Br J Dermatol 2019;180:373-81.

56. DeVries T, Ramachandram D. Skin lesion classification using deep multi-scale convolutional neural networks. arXiv preprint arXiv:170301402. 2017.

57. Mahbod A, Ecker R, Ellinger I. Skin lesion classification using hybrid deep neural networks. arXiv preprint arXiv:170208434. 2017.

58. Harangi B. Skin lesion classification with ensembles of deep convolutional neural networks. J Biomed Inform 2018;86:25-32.

59. Menegola A, Tavares J, Fornaciali M, Li LT, Avila S, Valle E. RECOD titans at ISIC challenge 2017. arXiv preprint arXiv:170304819. 2017.

60. Liu Y, Jain A, Eng C, Way DH, Lee K, Bui P, Kanada K, de Oliveira Marinho G, Gallegos J, Gabriele S, Gupta V, Singh N, Natarajan V, Hofmann-Wellenhof R, Corrado GS, Peng LH, Webster DR, Ai D, Huang SJ, Liu Y, Dunn RC, Coz D. A deep learning system for differential diagnosis of skin diseases. Nat Med 2020;26:900-8.

61. Osinga D. Deep Learning Cookbook: Practical Recipes to Get Started Quickly. 1st column. O’Reilly Media; 2018.

62. Redmon J, Farhadi A. Yolov3: An incremental improvement. arXiv preprintarXiv:180402767. 2018.

63. Kumar D, Hani AFM, editors. Optical imaging for biomedical and clinical applications. 1st editon. Boca Raton: CRC Press/Taylor \& Francis, 2018

64. Heintzmann R, Gustafsson MGL. Subdiffraction resolution in continuous samples. Nat Photonics 2009;3:362-4.

65. Kothari S, Phan JH, Wang MD. Eliminating tissuefold artifacts in histopathological whole-slide images for improved image-based prediction of cancer grade. J Pathol Inform 2013;4:22.

66. Khan AM, Rajpoot N, Treanor D, Magee D. A Nonlinear Mapping Approach to Stain Normalization in Digital Histopathology Images Using Image-Specific Color Deconvolution. IEEE Trans Biomed Eng 2014;61:1729-38.

67. Rubin R. Rubin's pathology: clinicopathologic foundations of medicine. Strayer DS, Rubin E, McDonald JM, Michalopoulos GK, editors. 5th edition. Lippincott 
Williams \& Wilkins, 2008

68. Vardiman JW. The World Health Organization (WHO) classification of tumors of the hematopoietic and lymphoid tissues: An overview with emphasis on the myeloid neoplasms. Chem Biol Interact 2010;184:16-20.

69. Shamir L, Orlov N, Mark Eckley D, Macura TJ, Goldberg IG. IICBU 2008: a proposed benchmark suite for biological image analysis. Med Biol Eng Comput 2008;46:943-7.

70. Sertel O, Kong J, Lozanski G, Arwa Sa, Catalyurek U, Saltz J, Gurcan M, editors. Texture classification using nonlinear color quantization: Application to histopathological image analysis. Proc IEEE Int Conf Acoust Speech Signal Process, 2008:507-600

71. Rawla P, Sunkara T, Barsouk A. Epidemiology of colorectal cancer: incidence, mortality, survival, and risk factors. Prz Gastroenterol 2019;14:89-103.

72. Linder N, Konsti J, Turkki R, Rahtu E, Lundin M, Nordling S, Haglund C, Ahonen T, Pietikäinen M, Lundin J. Identification of tumor epithelium and stroma in tissue microarrays using texture analysis. Diagn Pathol 2012;7:22.

73. Nava R, González G, Kybic J, Escalante-Ramírez B. Characterization of hematologic malignancies based on discrete orthogonal moments. 2016 Sixth International Conference on Image Processing Theory, Tools and Applications (IPTA). 12-15 Dec. 2016; Oulu, Finland. IEEE, 2016:1-6.

74. Flusser J, Suk T, Zitova B. 2D and 3D Image Analysis by Moments. John Wiley \& Sons, 2016.

75. González G, Nava R, Escalante-Ramírez B. A Comparative Study on Discrete Shmaliy Moments and Their TextureBased Applications. Math Probl Eng 2018;2018:1673283.

76. Marcos JV, Cristóbal G. Texture classification using discrete Tchebichef moments. J Opt Soc Am A Opt Image Sci Vis 2013;30:1580-91.

77. Mukundan R, Ong SH, Lee PA. Image analysis by Tchebichef moments. IEEE Trans Image Process 2001;10:1357-64.

78. Nava R, González G, Kybic J, Escalante-Ramírez B. Classification of Tumor Epithelium and Stroma in Colorectal Cancer Based on Discrete Tchebichef Moments. In: Laura CO, Shekhar R, Wesarg S, Gonzalez Ballester MÁ, Drechsler K, Sato Y, Erdt M, Linguraru MG. editors. Clinical Image-Based Procedures. Translational Research in Medical Imaging. CLIP 2015. Lecture Notes in Computer Science, vol 9401. Springer, Cham, 2016:78-87.
79. Huang Y, Zheng H, Liu C, Ding X, Rohde GK. Epithelium-Stroma Classification via Convolutional Neural Networks and Unsupervised Domain Adaptation in Histopathological Images. IEEE J Biomed Health Inform 2017;21:1625-32.

80. Yu X, Zheng H, Liu C, Huang Y, Ding X. Classify epithelium-stroma in histopathological images based on deep transferable network. J Microsc 2018;271:164-73.

81. Kather JN, Bello-Cerezo R, Di Maria F, van Pelt GW, Mesker WE, Halama N, Bianconi F. Classification of Tissue Regions in Histopathological Images: Comparison Between Pre-Trained Convolutional Neural Networks and Local Binary Patterns Variants. Nanni L, Brahnam S, Brattin R, Ghidoni S, Jain LC, editors. Deep Learners and Deep Learner Descriptors for Medical Applications 2020. Intelligent Systems Reference Library. Springer, 2020:95-115

82. Meng T, Lin L, Shyu ML, Chen SC, editors. Histology Image Classification Using Supervised Classification and Multimodal Fusion. 2010 IEEE International Symposium on Multimedia; 13-15 Dec. 2010; Taichung, Taiwan. IEEE, 2010:145-52.

83. Orlov NV, Chen WW, Eckley DM, Macura TJ, Shamir L, Jaffe ES, Goldberg IG. Automatic Classification of Lymphoma Images With Transform-Based Global Features. IEEE Trans Inf Technol Biomed 2010;14:1003-13.

84. Xu J, Luo X, Wang G, Gilmore H, Madabhushi A. A Deep Convolutional Neural Network for segmenting and classifying epithelial and stromal regions in histopathological images. Neurocomputing 2016;191:214-23.

85. Bianconi F, Bello-Cerezo R, Napoletano P. Improved opponent color local binary patterns: an effective local image descriptor for color texture classification. J Electron Imaging 2017;27:011002.

86. Song Y, Li Q, Huang H, Feng D, Chen M, Cai W. Low Dimensional Representation of Fisher Vectors for Microscopy Image Classification. IEEE Trans Med Imaging 2017;36:1636-49.

87. Luan S, Chen C, Zhang B, Han J, Liu J. Gabor Convolutional Networks. IEEE Trans Image Process 2018;27:4357-66.

88. Dolz J, Massoptier L, Vermandel M. Segmentation algorithms of subcortical brain structures on MRI for radiotherapy and radiosurgery: A survey. IRBM 2015;36:200-12.

89. González-Villà S, Oliver A, Valverde S, Wang L, Zwiggelaar R, Lladó X. A review on brain structures segmentation in magnetic resonance imaging. Artif Intell 
Med 2016;73:45-69.

90. Fischl B, Salat DH, Busa E, Albert M, Dieterich M, Haselgrove C, van der Kouwe A, Killiany R, Kennedy D, Klaveness S, Montillo A, Makris N, Rosen B, Dale AM. Whole brain segmentation: automated labeling of neuroanatomical structures in the human brain. Neuron 2002;33:341-55.

91. Scherrer B, Dojat M, Forbes F, Garbay C. LOCUS: LOcal Cooperative Unified Segmentation of MRI Brain Scans. Med Image Comput Comput Assist Interv 2007;10:219-27.

92. Carbajal-Degante E, Olveres J, Escalante-Ramírez B. A multiphase active contour model based on the Hermite transform for texture segmentation. Proceedings Volume 10679, Optics, Photonics, and Digital Technologies for Imaging Applications V; 106791H. 2018:364-72.

93. Jorge Cardoso M, Leung K, Modat M, Keihaninejad S, Cash D, Barnes J, Fox NC, Ourselin S; Alzheimer's Disease Neuroimaging Initiative. STEPS: Similarity and Truth Estimation for Propagated Segmentations and its application to hippocampal segmentation and brain parcelation. Med Image Anal 2013;17:671-84.

94. Pipitone J, Park MT, Winterburn J, Lett TA, Lerch JP, Pruessner JC, Lepage M, Voineskos AN, Chakravarty MM; Alzheimer's Disease Neuroimaging Initiative. Multi-atlas segmentation of the whole hippocampus and subfields using multiple automatically generated templates. Neuroimage 2014;101:494-512.

95. Patenaude B, Smith SM, Kennedy DN, Jenkinson M. A Bayesian model of shape and appearance for subcortical brain segmentation. NeuroImage 2011;56:907-22.

96. Gao Y, Corn B, Schifter D, Tannenbaum A. Multiscale 3D shape representation and segmentation with applications to hippocampal/caudate extraction from brain MRI. Med Image Anal 2012;16:374-85.

97. Li DH, He YC, Liu J, Chen SD. Diagnostic Accuracy of Transcranial Sonography of the Substantia Nigra in Parkinson's disease: A Systematic Review and Metaanalysis. Sci Rep 2016;6:20863

98. Olveres J, Carbajal-Degante E, Escalante-Ramírez B, Vallejo E, García-Moreno CM. Deformable Models for Segmentation Based on Local Analysis. Math Probl Eng 2017;2017:1646720.

99. Milletari F, Ahmadi SA, Kroll C, Plate A, Rozanski V, Maiostre J, et al. Hough-CNN: Deep learning for segmentation of deep brain regions in MRI and ultrasound. Comput Vis Image Underst 2017;164:92-102.

100. Kushibar K, Valverde S, González-Villà S, Bernal J, Cabezas M, Oliver A, Lladó X. Automated sub-cortical brain structure segmentation combining spatial and deep convolutional features. Med Image Anal 2018;48:177-86.

101. Perlmutter JS, Mink JW. Deep brain stimulation. Annu Rev Neurosci 2006;29:229-57.

102. WHO. Chronic obstructive pulmonary disease (COPD). World Health Organization: WHO. [Online; accessed: October 4th 2020]. Available online: https://www.who. $\mathrm{int} /$ news-room/fact-sheets/detail/chronic-obstructivepulmonary-disease-(copd)

103. Diab N, Gershon AS, Sin DD, Tan WC, Bourbeau J, Boulet LP, Aaron SD. Underdiagnosis and Overdiagnosis of Chronic Obstructive Pulmonary Disease. Am J Respir Crit Care Med 2018;198:1130-9.

104. Bibault JE, Xing L. Screening for chronic obstructive pulmonary disease with artificial intelligence. Lancet Digit Health 2020;2:e216-7.

105. Mekov E, Miravitlles M, Petkov R. Artificial intelligence and machine learning in respiratory medicine. Expert Rev Respir Med 2020;14:559-64.

106.Afzali A, Mofrad FB, Pouladian M. Contour-based lung shape analysis in order to tuberculosis detection: modeling and feature description. Med Biol Eng Comput 2020;58:1965-86.

107.Selvan R, Dam EB, Detlefsen NS, Rischel S, Sheng K, Nielsen M, Pai A. Lung Segmentation from Chest X-rays using Variational Data Imputation. Lung Segmentation from Chest X-rays using Variational Data Imputation. Presented at the first Workshop on the Art of Learning with Missing Values (Artemiss) hosted by the 37th International Conference on Machine Learning (ICML). Jul 2020.

108. Rajpurkar P, Irvin J, Zhu K, Yang B, Mehta H, Duan T, Ding D, Bagul A, Langlotz C, Shpanskaya K, Lungren MP, Ng AY. CheXNet: Radiologist-Level Pneumonia Detection on Chest X-Rays with Deep Learning. arXiv:1711.05225 Nov 2017.

109. Shi F, Wang J, Shi J, Wu Z, Wang Q, Tang Z, He K, Shi Y, Shen D. Review of Artificial Intelligence Techniques in Imaging Data Acquisition, Segmentation, and Diagnosis for COVID-19. IEEE Rev Biomed Eng 2021;14:4-15.

110.Zech JR, Badgeley MA, Liu M, Costa AB, Titano JJ, Oermann EK. Variable generalization performance of a deep learning model to detect pneumonia in chest radiographs: A cross-sectional study. PLoS Med 2018;15:e1002683.

111. Castillo-Saldana D, Hague CJ, Coxson HO, Ryerson CJ. Using Quantitative Computed Tomographic Imaging to Understand Chronic Obstructive Pulmonary Disease and 
Fibrotic Interstitial Lung Disease: State of the Art and

Future Directions. J Thorac Imaging 2020;35:246-54.

112.Lippi G, Henry BM. Chronic obstructive pulmonary disease is associated with severe coronavirus disease 2019 (COVID-19). Respir Med 2020;167:105941.

113.Li L, Qin L, Xu Z, Yin Y, Wang X, Kong B, Bai J, Lu Y, Fang Z, Song Q, Cao K, Liu D, Wang G, Xu Q, Fang X, Zhang S, Xia J, Xia J. Artificial Intelligence Distinguishes COVID-19 from Community Acquired Pneumonia on Chest CT. Radiology 2020;296:E65-71.

114.Le Berre C, Sandborn WJ, Aridhi S, Devignes MD, Fournier L, Smail-Tabbone M, Danese S, PeyrinBiroulet L. Application of Artificial Intelligence to Gastroenterology and Hepatology. Gastroenterology 2020;158:76-94.e2.

115.Kudo SE, Misawa M, Mori Y, Hotta K, Ohtsuka K, Ikematsu H, Saito Y, Takeda K, Nakamura H, Ichimasa $\mathrm{K}$, Ishigaki T, Toyoshima N, Kudo T, Hayashi T, Wakamura K, Baba T, Ishida F, Inoue H, Itoh $\mathrm{H}$, Oda $\mathrm{M}$, Mori K. Artificial Intelligence-assisted System Improves Endoscopic Identification of Colorectal Neoplasms. Clin Gastroenterol Hepatol 2020;18:1874-1881.e2.

116. Vivanti R, Szeskin A, Lev-Cohain N, Sosna J, Joskowicz L. Automatic detection of new tumors and tumor burden evaluation in longitudinal liver CT scan studies. Int J Comput Assist Radiol Surg 2017;12:1945-57.

117.Liu X, Song JL, Wang SH, Zhao JW, Chen YQ. Learning to Diagnose Cirrhosis with Liver Capsule Guided Ultrasound Image Classification. Sensors (Basel) 2017;17:149.

118. Biswas M, Kuppili V, Edla DR, Suri HS, Saba L, Marinhoe RT, Sanches JM, Suri JS. Symtosis: A liver ultrasound

Cite this article as: Olveres J, González G, Torres F, Moreno-Tagle JC, Carbajal-Degante E, Valencia-Rodríguez A, Méndez-Sánchez N, Escalante-Ramírez B. What is new in computer vision and artificial intelligence in medical image analysis applications. Quant Imaging Med Surg 2021;11(8):38303853. doi:10.21037/qims-20-1151 tissue characterization and risk stratification in optimized deep learning paradigm. Comput Methods Programs Biomed 2018;155:165-77.

119. Yasaka K, Akai H, Abe O, Kiryu S. Deep Learning with Convolutional Neural Network for Differentiation of Liver Masses at Dynamic Contrast-enhanced CT: A Preliminary Study. Radiology 2018;286:887-96.

120. Ibragimov B, Toesca D, Chang D, Koong A, Xing L. Combining deep learning with anatomical analysis for segmentation of the portal vein for liver SBRT planning. Phys Med Biol 2017;62:8943-58.

121. Mendez-Sanchez N, Cruz-Ramon VC, RamirezPerez OL, Hwang JP, Barranco-Fragoso B, CordovaGallardo J. New Aspects of Lipotoxicity in Nonalcoholic Steatohepatitis. Int J Mol Sci 2018;19:2034.

122.Méndez-Sánchez N, Chavez-Tapia NC, Almeda-Valdes P, Uribe $M$. The management of incidental fatty liver found on imaging. What do we need to do? Am J Gastroenterol 2018;113:1274-6.

123.Valencia-Rodríguez A, Vera-Barajas A, Barranco-Fragoso B, Kúsulas-Delint D, Qi X, Méndez-Sánchez N. New insights into the association between non-alcoholic fatty liver disease and atherosclerosis. Ann Transl Med 2019;7:S300.

124.López-Velázquez JA, Silva-Vidal KV, Ponciano-Rodríguez G, Chávez-Tapia NC, Arrese M, Uribe $M$ et al. The prevalence of nonalcoholic fatty liver disease in the Americas. Ann Hepatol 2014;13:166-78.

125.Zhou LQ, Wang JY, Yu SY, Wu GG, Wei Q, Deng YB, Wu XL, Cui XW, Dietrich CF. Artificial intelligence in medical imaging of the liver. World J Gastroenterol 2019;25:672-82. 